\title{
K- AND PAR POND SEWAGE SLUDGE APPLICATION SITES GROUNDWATER MONITORING REPORTS - SECOND QUARTER 1992
}

by WSRC Contact - C. Y. Thompson

Westinghouse Savannah River Company

Savannah River Site

Aiken, South Carolina 29808

Other Authors: $N^{\prime} \nmid$

This paper was prepared in connection with work done under Contract No. DE-AC09-89SR18035 with the U. S. Department of Energy. By acceptance of this paper, the publisher and/or recipient acknowledges the U. S. Government's right to retain a nonexclusive, royalty-free license in and to any copyright covering this paper, along with the right to reproduce and to authorize others to reproduce all or part of the copyrighted paper. 


\section{K-AREA AND PAR POND SEWAGE \\ SLUDGE APPLICATION SITES \\ GROUNDWATER MONITORING REPORT (U)}

KEY WORDS

alkalinity

chlordane

hazardous waste

KSS wells

PSS wells

PUBLICATION DATE: OCTOBER 1992

Authorized Derivative Classifier:

$m . q .96 m a$

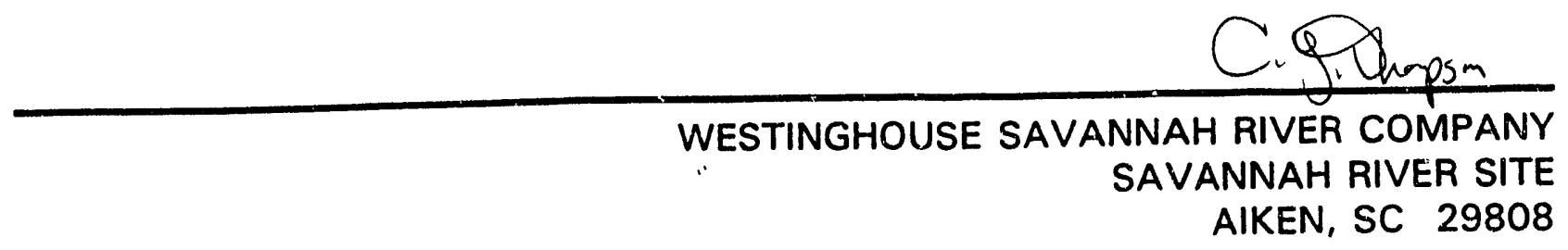




\section{Abstract}

During second quarter 1992, the three wells at the K-Area Sewage Sludge Application Site (KSS wells) and the three wells at the Par Pond Sewage Sludge Application Site (PSS wells) were sampled for analyses required each quarter or annually by South Carolina Department of Health and Environmental Control Construction Permit 13,173. None of the constituents analyzed for exceeded the Primary Drinking Water Standard or the Savannah River Site Flag 2 criteria at either the K-Area Sewage Sludge Application Site or the Par Pond Sewage Sludge Application Site. 


\section{Contents}

Page

Abstract $\ldots \ldots \ldots \ldots \ldots \ldots \ldots \ldots \ldots \ldots \ldots \ldots \ldots \ldots \ldots \ldots \ldots \ldots$

List of Figures $\ldots \ldots \ldots \ldots \ldots \ldots \ldots \ldots \ldots \ldots \ldots \ldots \ldots \ldots \ldots$ vii

List of Tables $\ldots \ldots \ldots \ldots \ldots \ldots \ldots \ldots \ldots \ldots \ldots \ldots \ldots \ldots \ldots \ldots \ldots$

Executive Summary $\ldots \ldots \ldots \ldots \ldots \ldots \ldots \ldots \ldots \ldots \ldots \ldots \ldots \ldots \ldots$

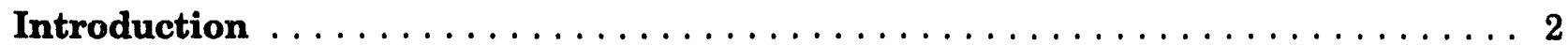

Discussion $\ldots \ldots \ldots \ldots \ldots \ldots \ldots \ldots \ldots \ldots \ldots \ldots \ldots \ldots \ldots \ldots \ldots \ldots$

Groundwater Monitoring Data $\ldots \ldots \ldots \ldots \ldots \ldots \ldots \ldots \ldots \ldots \ldots \ldots$

Analytical Results Exceeding Standards $\ldots \ldots \ldots \ldots \ldots \ldots \ldots \ldots \ldots$

Water Elevations and Groundwater Flow Directions $\ldots \ldots \ldots \ldots \ldots \ldots \ldots$

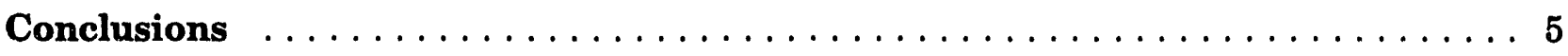

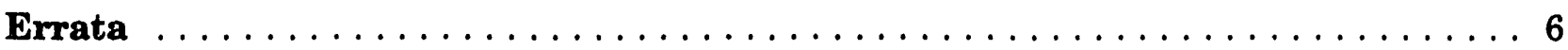

Appendix A-Primary Drinking Water Standards $\ldots \ldots \ldots \ldots \ldots \ldots \ldots$

Appendix B-Flagging Criteria $\ldots \ldots \ldots \ldots \ldots \ldots \ldots \ldots \ldots \ldots \ldots \ldots$

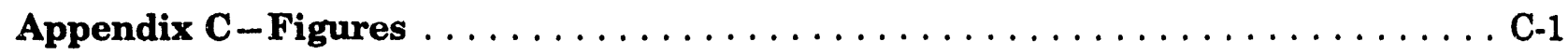

Appendix D-Groundwater Monitoring Results Tables . . . . . . . . . . . D-1 


\section{List of Figures}

Page

1. Location of the K-Area and Par Pond Sewage Sludge Application Sites

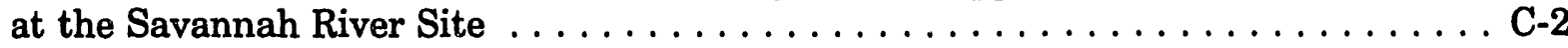

2. Location of Groundwater Monitoring Wells at the K-Area Sewage Sludge Application Site $\ldots \ldots \ldots \ldots \ldots \ldots \ldots \ldots \ldots \ldots \ldots \ldots$

3. Location of Groundwater Monitoring Wells at the Par Pond Sewage Sludge Application Site $\ldots \ldots \ldots \ldots \ldots \ldots \ldots \ldots \ldots \ldots \ldots$ C-4.

4. Potentiometric Surface Map of the K-Area Sewage Sludge Application Site . . . . . C-5

5. Potentiometric Surface Map of the Par Pond Sewage Sludge Application Site . . . . C-6 


\section{List of Tables}

Page

1. Constituents Exceeding the Primary Drinking Water Standards at the K-Area

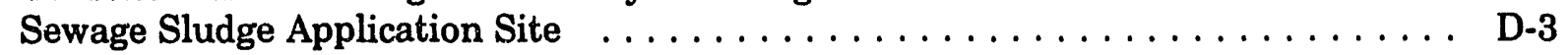

2. Constituents Exceeding the Primary Drinking Water Standards at the Par Pond Sewage Sludge Application Site $\ldots \ldots \ldots \ldots \ldots \ldots \ldots \ldots \ldots \ldots \ldots \ldots \ldots \ldots$

3. Constituents Exceeding Half the Primary Drinking Water Standards or Other Flag 1 or Flag 2 Criteria at the K-Area Sewage Sludge Application Site ...... D-4

4. Constituents Exceeding Half the Primary Drinking Water Standards or Other Flag 1 or Llagy 2 Criteria at the Par Pond Sewage Sludge Application Site ...... . D-4

5. Groundwater Monitoring Results for Individual Wells at the K-Area Sewage

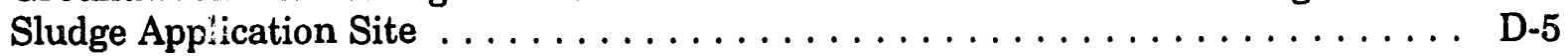

6. Groundwater Monitoring Results for Individual Wells at the Par Pond Sewage

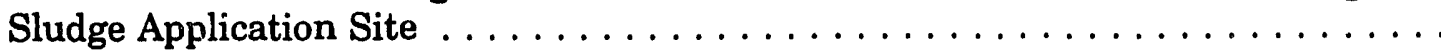




\section{Executive Summary}

Eleven sewage sludge application sites at the Savannah River Site (SRS) were originally the subject of a research program, begun in 1980, using domestic sewage sludge to reclaim borrow pits and to enhance forest productivity at SRS. As part of this research program, wastewater sludge was applied to the K-Area Sewage Sludge Application Site and Par Pond Sewage Sludge Application Site in 1981. These sites received a second and last application of sludge in 1988. This application was a one-time occurrence in accordance with Construction Permit 13,173, issued in April 1987 by the South Carolina Department of Health and Environmental Control (SCDHEC). Both sites are now inactive.

The monitoring wells at the K-Ares and Par Pond Sewage Sludge Application Sites are sampled quarterly as part of the SRS Goundwater Monitoring Program and to comply with SCDHEC Construction Permit 13,173.

During second quarter 1992, samples from the three monitoring wells at the K-Area site (KSS series) and the three monitoring wells at the Par Pond site (PSS series) were analyzed for constituents required by the SCDHEC construction permit and for other constituents required by the SRS Groundwater Monitoring Program. This report describes monitoring results that exceeded the U.S. Environmental Protection Agency Primary Drinking Water Standards (PDWS) or the SRS flagging criteria.

Well PSS 3D went dry during purging; thus, the sample collected from this well may not be a representative groundwater sample.

During second quarter 1992, none of the constituents analyzed for exceeded either the PDWS or the SRS Flag 2 criteria at the K-Area Sewage Sludge Application Site or the Par Pond Sewage Sludge Application Site.

The field measurement for alkalinity was as high as $9 \mathrm{mg} / \mathrm{L}$ in the KSS well series and 1 $\mathrm{mg} / \mathrm{L}$ in the PSS well series.

Historical water-level elevations at the K-Area Sewage Sludge Application Site and at the Par Pond Sewage Sludge Application Site indicate that the groundwater flow directions are south to southwest (SRS grid coordinates). Thus, at the K-Area Sewage Sludge Application Site, well KSS $1 \mathrm{D}$ is upgradient, KSS 3D is downgradient, and KSS 2D is downgradient within the southern border of the application area. At the Par Pond Sewage Sludge Application Site, well PSS 1D is upgradient, and wells PSS 2D and 3D are downgradient. 


\section{Introduction}

The 11 sewage sludge application sites at the Savannah River Site (SRS) were originally the subject of a research program, begun in 1980, using domestic sewage sludge to reclaim borrow pits and to enhance forest productivity at SRS. After sludge was applied to the sites, hardwoods and pines were planted to quantify the wood biomass that could be produced using the sludge as a fertilizer and soil conditioner. As pari of this research program, wastewater sludge was applied to the K-Area Sewage Sludge Application Site and Par Pond Sewage Sludge Application Site in 1981 under Industrial Waste Permit 175.

The 17-acre K-Area Sewage Sludge Application Site lies southeast of K Area on the west bank of Pen Branch (see Figures 1 and 2 in Appendix C). The Par Pond Sewage Sludge Application Site includes 22 acres south of Par Pond, approximately 1.3 miles north-northeast of the intersection of Roads B and F (see Figures 1 and 3 in Appendix C). Both sites are now inactive. They received a second and last application of sludge in 1988. This application, from the Central Shops Sanitary Sludge Lagoon, was a one-time occurrence in accordance with Construction Permit 13,173, issued in April 1987 by the South Carolina Department of Health and Environmental Control (SCDHEC).

In 1988, SRS determined that new wells were required at the K-Area and Par Pond Sewage Sludge Application Sites to assess the effects of sewage sludge application on groundwater. SRS installed three new wells at the K-Area site and three new wells at the Par Pond site after approval by SCDHEC. These wells, which were first sampled during fourth quarter 1988, are designated KSS 1D, 2D, and 3D and PSS 1D, 2D, and 3D. All wells monitor the water table and are constructed of 4-in. polyvinyl chloride pipe.

In 1989, the K-Arca and Par Pond Sewage Sludge Application Sites became part of the Resource Conservation and Recovery Act Facilities Investigation/Remedial Investigation program because chlordane was found in the sludge from the Central Shops lagoon.

Data from the six wells monitoring the K-Area and Par Pond Sewage Sludge Application Sites for second quarter 1992 are presented in this report as required by Special Condition 4 of SCDHEC Construction Permit 13,173. 


\section{Discussion}

\section{Groundwater Monitoring Data}

The standard practice at SRS is to purge a minimum of four well volumes from each well and ensure that the field parameters have stabilized before sample collection. A well that goes dry before completion of purging is allowed to recover and is sampled without further purging within 24 hours. If successful, this sequence constitutes a single sampling event. During second quarter 1992, well PSS 3D went dry during purging; thus, the sample collected from this well may not be a representative groundwater sample. PSS 3D has consist y purged dry since its installation.

Samples from the three monitoring wells at the K-Area Sewage Sludge Application Site and the three monitoring wells at the Par Pond Sewage Sludge Application Site are analyzed quarterly for the following parameters as required by SCDHEC Construction Permit 13,173:

- Specific conductance and $\mathrm{pH}$ (laboratory measurements)

- Water quality indicators: chloride, nitrate and nitrite (as nitrogen), sodium, and total dissolved solids

Annually, these wells are also analyzed for cadmium, calcium, copper, iron, lead, magnesium, manganese, nickel, potassium, and total phosphates (as phosphorus) as required by the construction permit. The wells may also receive additional analyses as part of the SRS Groundwater Monitoring Program.

This report describes monitoring results that exceeded the U.S. Environmental Protection Agency Primary Drinking Water Standards (PDWS) (see Appendix A) or the SRS flagging criteria (see Appendix B) during second quarter 1992.

Appendix $\mathrm{C}$ shows the locations of the monitored sites at SRS (Figure 1.), the individual KSS and PSS monituring wells (Figures 2 and 3, respectively), and the flow directions of the groundwater beneath the sites (Figures 4 and 5). Monitoring results tables as well as analyses that exceeded the holding times and PDWS are in Appendix D.

\section{Analytical Results Exceeding Standards}

Tables 1 and 2 (Appendix D) summarize any constituents that exceeded the PDWS at the K-Area Sewage Sludge Application Site or Par Pond Sewage Sludge Application Site, respectively, during second quarter 1992. None of the constituents analyzed for exceeded the PDWS in the KSS or PSS wells.

Constituents exceeding other flagging criteria (see Appendix B) during second quarter 1992 are summarized in Table 3 (Appendix D) for the K-Area Sewage Sludge Application Site 
and Table 4 (Appendix D) for the Par Pond Sewage Sludge Application Site. None of the constituents analyzed for exceeded the SRS Flag 2 criteria at either site.

Results are presented for all analyzed constituents for the KSS and PSS wells in Tables 5 and 6, respectively (Appendix D).

Presently, SRS sets no flagging criteria for alkalinity. In the KSS well series, alkalinity ranged as high as $9 \mathrm{mg} / \mathrm{L}$ in well $\mathrm{KSS} 3 \mathrm{D}$. Alkalinity reached $1 \mathrm{mg} / \mathrm{L}$ in wells PSS $1 \mathrm{D}$ and $3 D$.

\section{Water Elevations and Groundwater Flow Directions}

Water-level elevations at the K-Area Sewage Sludge Application Site indicate that the groundwater flow direction is generally south to southwest (SRS coordinates), although the nearly linear orientation of these wells mates such a determination tentative. Well KSS ID is upgradient, KSS $3 \mathrm{D}$ is downgradient, and KSS 2D is downgradient within the southern border of the application area.

During second quarter 1992, only two water-level elevations were obtained at the Par Pond Sewage Sludge Application Site. Thus, determination of flow direction was not possible. Historically, flow direction has been reported as south to southwest (SRS coordinates). Based upon this precedent, well PSS $1 D$ is upgradient, and wells PSS 2D and 3D are cowngradient. 


\section{Conclusions}

- During second quarter 1992, none of the constituents analyzed exceeded either the PDWS or the SRS Flag 2 criteria at the K-Area Sewage Sludge Application Site.

- During second quarter 1992, none of the constituents analyzed exceeded either the PDWS or the SRS Flag 2 criteria at the Par Pond Sewage Sludge Application Site. 


\section{Errata}

First Quarter 1992:

- Chlordane analysis was requested as part of the Base/Neutral/Acid suite of analyses as described in the Environmental Protection Department/Environmental Monitoring Section contract with the analytical laboratory. However, the Roy F. Weston laboratory, who conducted the analyses for first through third quarters 1992 , does not include chlordane in their Base/Neutral/Acid suite of analyses. Chlordane analysis will be conducted by General Engineering Laboratories for fourth quarter 1992. 


\section{Appendix A - Primary Drinking Water Standards}

(2) 


\section{Primary Drinking Water Standards}

\begin{tabular}{|c|c|c|c|c|}
\hline Analyte & Unit & Level & Status & Reference \\
\hline Americium-241 & $\mathrm{pCi} / \mathrm{L}$ & $6.34 E+00$ & Proposed & EPA, 1991 \\
\hline Americium-243 & $\mathrm{pCi} / \mathrm{L}$ & $6.37 E+00$ & Proposed & EPA, 1991 \\
\hline Antimony & $\mu g / L$ & $5^{a}$ & Proposed & EPA, 1990 \\
\hline Antimony-125 & $\mathrm{pCi} / \mathrm{L}$ & $3 E+02$ & Final & EPA, 1977 \\
\hline Arsenic & $\mu \mathrm{g} / \mathrm{L}$ & 50 & Final & CFR, 1991 \\
\hline Barium & $\mu \mathrm{g} / \mathrm{L}$ & 2,000 & Final & CFR, 1991 \\
\hline Barium-140 & $\mathrm{pCi} / \mathrm{L}$ & $9 E+01$ & Final & EPA, 1977 \\
\hline Benzene & $\mu g / L$ & 5 & Final & CFR, 1991 \\
\hline Benzola]anthracene & $\mu g / L$ & 0.1 & Proposed & EPA, 1990 \\
\hline Benzo[b]fluoranthene & $\mu g / L$ & 0.2 & Proposed & EPA, 1990 \\
\hline Benzo[k]fluoranthene & $\mu g / L$ & 0.2 & Proposed & EPA, 1990 \\
\hline Benzolalpyrene & $\mu g / L$ & 0.2 & Proposed & EPA, 1990 \\
\hline Beryllium & $\mu g / L$ & 1 & Proposed & EPA, 1990 \\
\hline Beryllium-7 & $\mathrm{pCi} / \mathrm{L}$ & $6 E+03$ & Final & EPA, 1977 \\
\hline Bromodichloromethane & $\mu g / L$ & 100 & Final & CFR, 1991 \\
\hline Bromoform & $\mu \mathrm{g} / \mathrm{L}$ & 100 & Final & CFR, 1991 \\
\hline 2-sec-Butyl-4,6-dinitrophenol (Dinoseb) & $\mu \mathrm{g} / \mathrm{L}$ & 7 & Proposed & EPA, 1990 \\
\hline Cadmium & $\mu g / L$ & 5 & Final & CFR, 1991 \\
\hline Carbon-14 & $\mathrm{pCi} / \mathrm{L}$ & $2 E+03$ & Final & EPA, 1977 \\
\hline Carbon tetrachloride & $\mu g / L$ & 5 & Final & CFR, 1991 \\
\hline Cerium-141 & $\mathrm{pCi} / \mathrm{L}$ & $3 E+02$ & Final & EPA, 1977 \\
\hline Cerium-144 & $\mathrm{pCi} / \mathrm{L}$ & $2.61 E+02$ & Proposed & EPA, 1991 \\
\hline Cesium-134 & $\mathrm{pCi} / \mathrm{L}$ & $8.13 E+02^{b}$ & Proposed & EPA, 1991 \\
\hline Cesium-137 & $\mathrm{pCi} / \mathrm{L}$ & $2 E+02$ & Final & EPA, 1977 \\
\hline Chlordane & $\mu g / L$ & 2 & Final & CFR, 1991 \\
\hline Chloroethene (Vinyl chloride) & $\mu g / L$ & 2 & Final & CFR, 1991 \\
\hline Chloroform & $\mu \mathrm{g} / \mathrm{L}$ & 100 & Final & CFR, 1991 \\
\hline Chromium & $\mu \mathrm{g} / \mathrm{L}$ & 100 & Final & CFR, 1991 \\
\hline Chromium-51 & $\mathrm{pCi} / \mathrm{L}$ & $6 E+03$ & Final & EPA, 1977 \\
\hline Chrysene & $\mu g / L$ & 0.2 & Proposed & EPA, 1990 \\
\hline Cobalt-57 & $\mathrm{pCi} / \mathrm{L}$ & $1 E+03$ & Final & EPA, 1977 \\
\hline Cobalt-58 & $\mathrm{pCi} / \mathrm{L}$ & $9 E+03$ & Final & EPA, 1977 \\
\hline Cobalt -60 & $\mathrm{pCi} / \mathrm{L}$ & $1 E+02$ & Final & EPA, 1977 \\
\hline Copper & $\mu g / L$ & 1,300 & Final & CFR, 1991 \\
\hline Curium-242 & $\mathrm{pCi} / \mathrm{L}$ & $1.33 E+02$ & Proposed & EPA, 1991 \\
\hline Curium-243 & $\mathrm{pCi} / \mathrm{L}$ & $8.3 E+00$ & Proposed & EPA, 1991 \\
\hline Curium-244 & $\mathrm{pCi} / \mathrm{L}$ & $9.84 E+00$ & Proposed & EPA, 1991 \\
\hline Curium-246 & $\mathrm{pCi} / \mathrm{L}$ & $6.27 E+00$ & Proposed & EPA, 1991 \\
\hline Cyanide & $\mu g / L$ & 200 & Proposed & EPA, 1990 \\
\hline Dibenz $[a, h]$ lanthracene & $\mu g / L$ & 0.3 & Proposed & EPA, 1990 \\
\hline Dibromochloromethane & $\mu g / L$ & 100 & Final & CFR, 1991 \\
\hline Dibromochloropropane & $\mu \mathrm{g} / \mathrm{L}$ & 0.2 & Final & CFR, 1991 \\
\hline 1,2-Dichlorobenzene & $\mu g / L$ & 600 & Final & CFR, 1991 \\
\hline 1,4-Dichlorobenzene & $\mu g / L$ & 75 & Final & CFR, 1991 \\
\hline 1,2-Dichloroethane & $\mu g / L$ & 5 & Final & CFR, 1991 \\
\hline 1,1-Dichloroethylene & $\mu g / L$ & 7 & Final & CFR, 1991 \\
\hline cis-1,2-Dichloroethylene & $\mu g / L$ & 70 & Final & CFR, 1991 \\
\hline trans-1,2-Dichloroethylene & $\mu \mathrm{g} / \mathrm{L}$ & 100 & Final & CFR, 1991 \\
\hline Dichloromethane (Methylene chloride) & $\mu \mathrm{g} / \mathrm{L}$ & 5 & Proposed & EPA, 1990 \\
\hline 2,4-Dichlorophenoxyacetic acid & $\mu \mathrm{g} / \mathrm{L}$ & 70 & Final & CFR, 1991 \\
\hline 1,2-Dichloropropane & $\mu g / L$ & 5 & Final & CFR, 1991 \\
\hline Endrin & $\mu \mathrm{g} / \mathrm{L}$ & 0.2 & Final & CFR, 1991 \\
\hline Ethylbenzene & $\mu \mathrm{g} / \mathrm{L}$ & 700 & Final & CFR, 1991 \\
\hline Europium-154 & $\mathrm{pCi} / \mathrm{L}$ & $2 E+02$ & Final & EPA, 1977 \\
\hline
\end{tabular}




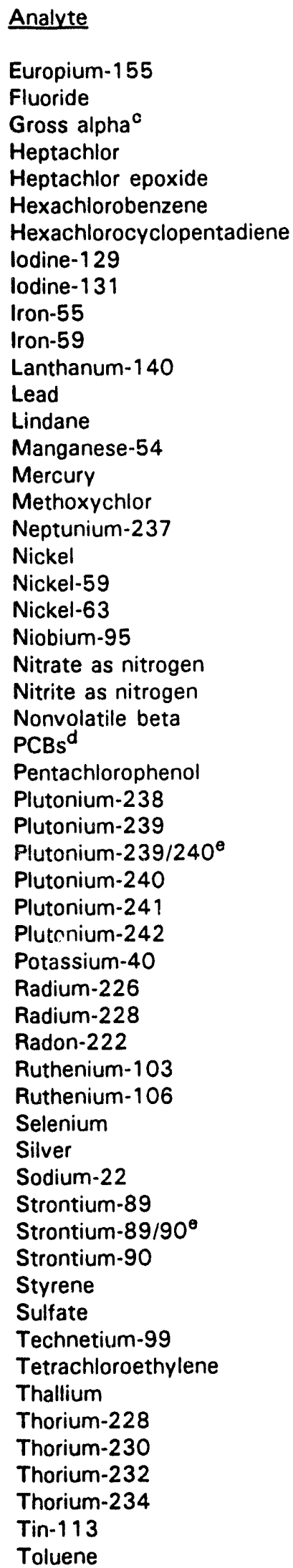

\begin{tabular}{|c|c|c|c|}
\hline Unit & Level & $\underline{\text { Status }}$ & Reference \\
\hline$p C i / L$ & $6 E+02$ & Final & EPA, 1977 \\
\hline$\mu g / L$ & 4,000 & Final & CFR, 1991 \\
\hline $\mathrm{pCi} / \mathrm{L}$ & $1.5 E+01$ & Final & CFR, 1991 \\
\hline$\mu g / L$ & 0.4 & Final & CFR, 1991 \\
\hline$\mu g / L$ & 0.2 & Final & CFR, 1991 \\
\hline$\mu \mathrm{g} / \mathrm{L}$ & 1 & Proposed & EPA, 1990 \\
\hline$\mu g / L$ & 50 & Proposed & EPA, 1990 \\
\hline $\mathrm{pCi}$ & $1 E+00$ & Final & EPA, 1977 \\
\hline $\mathrm{pCi} / \mathrm{L}$ & $3 E+00$ & Final & EPA, 197? \\
\hline $\mathrm{pCi} / \mathrm{L}$ & $2 E+03$ & Final & EPA, 1977 \\
\hline $\mathrm{pCi} / \mathrm{L}$ & $2 E+02$ & Final & EPA, 1977 \\
\hline $\mathrm{pCi} / \mathrm{L}$ & $6 \mathrm{E}+01$ & Final & EPA, 1977 \\
\hline$\mu g / L$ & 15 & Final & CFR, 1991 \\
\hline$\mu g / L$ & 0.2 & Final & CFR, 1991 \\
\hline $\mathrm{pCi} / \mathrm{L}$ & $3 E+02$ & Final & EPA, 1977 \\
\hline$\mu g / L$ & 2 & Final & CFR, 1991 \\
\hline$\mu g / L$ & 40 & Final & CFR, 1991 \\
\hline$p \mathrm{Ci} / \mathrm{L}$ & $7.06 E+00$ & Proposed & EPA, 1991 \\
\hline$\mu \mathrm{g} / \mathrm{L}$ & 100 & Proposed & EPA, 1990 \\
\hline $\mathrm{pCi} / \mathrm{L}$ & $3 E+02$ & Final & EPA, 1977 \\
\hline $\mathrm{pCi} / \mathrm{L}$ & $5 E+01$ & Final & EPA, 1977 \\
\hline $\mathrm{pCi} / \mathrm{L}$ & $3 E+02$ & Final & EPA, 1977 \\
\hline$\mu \mathrm{g} / \mathrm{L}$ & 10,000 & Final & CFR, 1991 \\
\hline$\mu g / L$ & 1.000 & Final & CFR, 1991 \\
\hline $\mathrm{pCi} / \mathrm{L}$ & $5 E+01$ & Proposed & EPA, 1986 \\
\hline$\mu \mathrm{g} / \mathrm{L}$ & 0.5 & Final & CFR, 1991 \\
\hline$\mu \mathrm{g} / \mathrm{L}$ & 1 & Final & CFR, 1991 \\
\hline $\mathrm{pCi} / \mathrm{L}$ & $7.02 E+00$ & Proposed & EPA, 1991 \\
\hline $\mathrm{pCi} / \mathrm{L}$ & $6.21 E+01$ & Proposed & EPA, 1991 \\
\hline $\mathrm{pCi} / \mathrm{L}$ & $6.21 E+01$ & Proposed & EPA, 1991 \\
\hline $\mathrm{pCi} / \mathrm{L}$ & $6.22 E+01$ & Proposed & EPA, 1991 \\
\hline $\mathrm{pCi} / \mathrm{L}$ & $6.26 E+01$ & Proposed & EPA, 1991 \\
\hline $\mathrm{pCi} / \mathrm{L}$ & $6.54 E+01$ & Proposed & EPA, 1991 \\
\hline $\mathrm{pCi} / \mathrm{L}$ & $3 E+02$ & Proposed & EPA, 1986 \\
\hline $\mathrm{pCi} / \mathrm{L}$ & $1.57 E+01$ & Proposed & EPA, 1991 \\
\hline $\mathrm{pCi} / \mathrm{L}$ & $7.85 E+00$ & Proposed & EPA, 1991 \\
\hline $\mathrm{pCi} / \mathrm{L}$ & $3 E+02$ & Proposed & EPA, 1991 \\
\hline pCi/L & $2 E+02$ & Final & EPA, 1977 \\
\hline $\mathrm{pCi} / \mathrm{L}$ & $3 E+01$ & Final & EPA, 1977 \\
\hline$\mu \mathrm{g} / \mathrm{L}$ & 50 & Final & CFR, 1991 \\
\hline$\mu g / L$ & 50 & Final & CFR, 1991 \\
\hline $\mathrm{pCi} / \mathrm{L}$ & $4.66 E+02$ & Proposed & EPA, 1991 \\
\hline $\mathrm{pCi} / \mathrm{L}$ & $2 E+01^{f}$ & Final & EPA, 1977 \\
\hline $\mathrm{pCi} / \mathrm{L}$ & $8 E+00$ & Final. & CFR, 1991 \\
\hline $\mathrm{pCi} / \mathrm{L}$ & $8 E+00$ & Final & CFR, 1991 \\
\hline$\mu g / L$ & 100 & Final & CFR, 1991 \\
\hline$\mu \mathrm{g} / \mathrm{L}$ & $400,000^{a}$ & Proposed & EPA, 1990 \\
\hline $\mathrm{pCi} / \mathrm{L}$ & $9 E+02$ & Final & EPA, 1977 \\
\hline$\mu \mathrm{g} / \mathrm{L}$ & 5 & Final & CFR, 1991 \\
\hline$\mu \mathrm{g} / \mathrm{L}$ & 1 & Proposed & EPA, 1990 \\
\hline $\mathrm{pCi} / \mathrm{L}$ & $1.25 E+02$ & Proposed & EPA, 1991 \\
\hline $\mathrm{pCi} / \mathrm{L}$ & $7.92 E+01$ & Proposed & EPA, 1991 \\
\hline $\mathrm{pCi} / \mathrm{L}$ & $8.8 E+01$ & Proposed & EPA, 1991 \\
\hline $\mathrm{pCi} / \mathrm{L}$ & $4.01 E+02$ & Proposed & EPA, 1991 \\
\hline$\rho \mathrm{Ci} / \mathrm{L}$ & $3 E+02$ & Final & EPA, 1977 \\
\hline$\mu g / L$ & 1,000 & Final & CFR, 1991 \\
\hline
\end{tabular}


Analyte

Total radium

Total trihalomethanes

Toxaphene

2,4,5-TP (Silvex)

1,2,4-Trichlorobenzene

1,1,1-Trichloroethane

1,1,2-Trichloroethane

Trichloroethylene

Tritium

Uranium

Uranium alpha activity

Uranium-233/234 ${ }^{\circ}$

Uranium-234

Uranium-235

Uranium-238

Xylenes

Zinc-65

Zirconium-95

Zirconium/Niobium-95

$\begin{array}{llll}\text { Unit } & \text { Level } & \text { Status } & \text { Reference } \\ \mathrm{pCi} / \mathrm{L} & 5 \mathrm{E}+00 & \text { Final } & \text { CFR, 1991 } \\ \mu \mathrm{g} / \mathrm{L} & 100 & \text { Final } & \text { CFR, 1991 } \\ \mu \mathrm{g} / \mathrm{L} & 3 & \text { Final } & \text { CFR, 1991 } \\ \mu \mathrm{g} / \mathrm{L} & 50 & \text { Final } & \text { CFR, 1991 } \\ \mu \mathrm{g} / \mathrm{L} & 9 & \text { Proposed } & \text { EPA, 1990 } \\ \mu \mathrm{g} / \mathrm{L} & 200 & \text { Final } & \text { CFR, 1991 } \\ \mu \mathrm{g} / \mathrm{L} & 5 & \text { Proposed } & \text { EPA, 1990 } \\ \mu \mathrm{g} / \mathrm{L} & 5 & \text { Final } & \text { CFR, 1991 } \\ \mathrm{pCi} / \mathrm{mL} & 2 \mathrm{E}+01 & \text { Final } & \text { CFR, 1991 } \\ \mu \mathrm{g} / \mathrm{L} & 20 & \text { Proposed } & \text { EPA, 1991 } \\ \mathrm{pCi} / \mathrm{L} & 3 E+01 & \text { Proposed } & \text { EPA, 1991 } \\ \mathrm{pCi} / \mathrm{L} & 1.38 E+01 & \text { Proposed } & \text { EPA, 1991 } \\ \mathrm{pCi} / \mathrm{L} & 1.39 E+01 & \text { Proposed } & \text { EPA, 1991 } \\ \mathrm{pCi} / \mathrm{L} & 1.45 E+01 & \text { Proposed } & \text { EPA, 1991 } \\ \mathrm{pCi} / \mathrm{L} & 1.46 E+01 & \text { Proposed } & \text { EPA, 1991 } \\ \mu \mathrm{g} / \mathrm{L} & 10,000 & \text { Final } & \text { CFR, 1991 } \\ \mathrm{pCi} / \mathrm{L} & 3 E+02 & \text { Final } & \text { EPA, 1977 } \\ \mathrm{pCi} / \mathrm{L} & 2 \mathrm{E}+02 & \text { Final } & \text { EPA, 1977 } \\ \mathrm{pCi} / \mathrm{L} & 2 \mathrm{E}+02 & \text { Final } & \text { EPA, 1977 }\end{array}$

Note: Drinking water standards set by EPA (1977) correspond to the level at which each radionuclide contributes $4 \mathrm{mrem} / \mathrm{yr}$ of dose to an individual consuming $2 \mathrm{~L}$ of contaminated liquid a day. See EPA (1977) for details.

a This value is the lower of two proposed levels.

b EPD/EMS uses the proposed standard because it is a lower value; the final PDWS in 1977 may have been in error.

c The standard given is for gross alpha including radium-226 but excluding radon and uranium.

d Analyses were conducted in 1992 for the following: PCB 1016, PCB 1221, PCB 1232, PCB 1242, PCB 1248, PCB 1254, and PCB 1260.

- For double radionuclide analyses where each separate radionuclide has its own standard, the more stringent standard is used.

$f$ This value is the lower of two levels given for strontium-89.

\section{References}

CFR (Code of Federal Regulations), 1991. National Psimary Drinking Water Regulations, 40 CFR, Part 141, pp. 578-715. Washington, DC.

EPA (U.S. Environmental Protection Agency), 1977. National Interim Primary Drinking Water Regulations, EPA-570/9-76-003. Washington, DC.

EPA (U.S. Environmental Protection Agency), 1986. Water Pollution Control; National Primary Drinking Water Regulations, Radionuclides (Proposed), Federal Register, September 30, 1986, pp. 34835-34862. Washington, DC.

EPA (U.S. Environmental Protection Agency), 1990. National Primary and Secondary Drinking Water Regulations; Synthetic Organic Chemicals and Inorganic Chemicals; Proposed Rule, Federal Register, July 25, 1990, pp. 30369-30448. Washington, DC.

EPA (U.S. Environmental Protection Agency), 1991. National Primary Drinking Water Regulations; Radionuclides; Proposed Rule, Federal Register, July 18, 1991, pp. 33052-33127. Washington, DC. 


\section{Appendix B - Flagging Criteria}




\section{Flagging Criteria}

Beginning in 1991, the Environmental Monitoring Section of the Environmental Protection Department (EPD/EMS) modified its guidelines for flagging constituents in the Groundwater Monitoring Program. These flagging criteria are as follows:

- Flag 2 criteria for constituents equal the U.S. Environmental Protection Agency (EPA) Primary Drinking Water Standard, the EPA proposed Primary Drinking Water Standard, or the EPA Secondary Drinking Water Standard. If a constituent does not have a drinking water standard, the Flag 2 criterion equals 10 times the method detection limit (MDL) calculated as the 90 th percentile detection limit obtained recently by one of the primary analytical laboratories.

- Flag 1 criteria for constituents equal one-half of the EPA Primary Drinking Water Standard, one-half the EPA proposed Primary Drinking Water Standard, or one-half the EPA Secondary Drinking Water Standard. If a constituent does not have an EPA drinking water standard, the Flag 1 criterion equals 5 times the MDL calculated as the 90 th percentile detection limit obtained recently by one of the primary analytical laboratories.

- Analytical results for constituents below Flag 1 or below sample detection limits are classified as Flag 0.

The following parameters are not assigned flagging criteria: alkalinity, calcium, carbonate, color, corrosivity, magnesium, odor, potassium, Eh, silica, sodium, total dissolved solids, total phosphorus, total phosphates (as P), and turbidity. In addition, common laboratory contaminants and cleaners including phthalates, methylene chloride, ketones, and toluene are not assigned flagging criteria.

Analyte
Acenaphthene
Acenaphthylene
Acetone
Acetonitrile (Methyl cyanide)
Acetophenone
2-Acetylaminofluorene
Acrolein
Acrylonitrile
Aldrin
Alkalinity (as CaCO 3 )
Allyl chloride
Aluminum
Americium-241
Americium-243
$4-$ Aminobiphenyl
Ammonia
Amrnonia nitrogen
Aniline
Anthracene
Antimony
Antimony-125

Analyte

Acetone

Acetonitrile (Methyi cyanide)

Acrolein

Acrylonitrile

Alkalinity (as $\mathrm{CaCO}_{3}$ )

Allyl chloride

Americium-241

Americium-243

4-Aminobiphenyl

Aniline

Antimony-125

\begin{tabular}{|c|c|c|}
\hline Unit & Flag 1 & Flag 2 \\
\hline$\mu g / L$ & 50 & 100 \\
\hline$\mu \mathrm{g} / \mathrm{L}$ & 50 & 100 \\
\hline$\mu \mathrm{g} / \mathrm{L}$ & 50 & 100 \\
\hline$\mu \mathrm{g} / \mathrm{L}$ & 500 & 1,000 \\
\hline$\mu \mathrm{g} / \mathrm{L}$ & 50 & 100 \\
\hline$\mu g / L$ & 50 & 100 \\
\hline$\mu \mathrm{g} / \mathrm{L}$ & 100 & 200 \\
\hline$\mu \mathrm{g} / \mathrm{L}$ & 100 & 200 \\
\hline$\mu \mathrm{g} / \mathrm{L}$ & 2.5 & 5 \\
\hline & No flag & No flag \\
\hline$\mu g / L$ & 250 & 500 \\
\hline$\mu \mathrm{g} / \mathrm{L}$ & 100 & 200 \\
\hline $\mathrm{pCi} / \mathrm{L}$ & $3.17 E+00$ & $6.34 E+00$ \\
\hline $\mathrm{pCi} / \mathrm{L}$ & $3.19 E+00$ & $6.37 E+00$ \\
\hline$\mu g / L$ & 50 & 100 \\
\hline$\mu g / L$ & 500 & 1,000 \\
\hline$\mu g / L$ & 50 & 100 \\
\hline$\mu g / L$ & 50 & 100 \\
\hline$\mu g / L$ & 50 & 100 \\
\hline$\mu \mathrm{g} / \mathrm{L}$ & 2.5 & 5 \\
\hline $\mathrm{pCi} / \mathrm{L}$ & $1.5 E+02$ & $3 E+02$ \\
\hline
\end{tabular}

Source

EPA Method 8270

EPA Method 8270

EPA Method 8240

EPA Method 8240

EPA Method 8270

EPA Method 8270

EPA Method 8240

EPA Method 8240

EPA Method 8080

Set by EPD/EMS

EPA Method 8240

EPA Method 6010

Proposed DWS (EPA, 1991)

Proposed DWS (EPA, 1991)

EPA Method 8270

APHA Method 417B

EPA Method 350.1

EPA Method 8270

EPA Method 8270

Proposed DWS (EPA, 1990)

Final DWS (EPA, 1977) 


\begin{tabular}{|c|c|c|c|c|}
\hline Analyte & Unit & Flag 1 & Flag 2 & Source \\
\hline Aramite & $\mu g / L$ & 50 & 100 & EPA Method 8270 \\
\hline Arsenic & $\mu \mathrm{g} / \mathrm{L}$ & 25 & 50 & Final DWS (CFR, 1991a) \\
\hline Azobenzene & $\mu g / L$ & 50 & 100 & EPA Method 625 \\
\hline Barium & $\mu \mathrm{g} / \mathrm{L}$ & 1,000 & 2,000 & Final DWS (CFR, 1991a) \\
\hline Barium-140 & $\mathrm{pCi} / \mathrm{L}$ & $4.5 E+01$ & $9 E+01$ & Final DWS (EPA, 1977) \\
\hline Benzene & $\mu \mathrm{g} / \mathrm{L}$ & 2.5 & 5 & Final DWS (CFR, 1991a) \\
\hline alpha-Benzene hexachloride & $\mu g / L$ & 2.5 & 5 & EPA Method 8080 \\
\hline beta-Benzene hexachloride & $\mu \mathrm{g} / \mathrm{L}$ & 2.5 & 5 & EPA Method 8080 \\
\hline delta-Benzene hexachloride & $\mu \mathrm{g} / \mathrm{L}$ & 2.5 & 5 & EPA Method 8080 \\
\hline Benzidine & $\mu \mathrm{g} / \mathrm{L}$ & 250 & 500 & EPA Method 8270 \\
\hline Benzolalanthracene & $\mu \mathrm{g} / \mathrm{L}$ & 0.05 & 0.1 & Proposed DWS (EPA, 1990) \\
\hline Benzolb]fluoranthene & $\mu \mathrm{g} / \mathrm{L}$ & 0.1 & 0.2 & Proposed DWS (EPA, 199C) \\
\hline Benzolk]fluoranthene & $\mu g / L$ & 0.1 & 0.2 & Proposed DWS (EPA, 19S0) \\
\hline Benzolg,h,i]perylene & $\mu g / L$ & 50 & 100 & EPA Method 8270 \\
\hline Benzolalpyrene & $\mu \mathrm{g} / \mathrm{L}$ & 0.1 & 0.2 & Pruposed DWS (EPA, 1990 ) \\
\hline Benzoic acid & $\mu g / L$ & 250 & 500 & EPA Method 8270 \\
\hline 1,4-Benzoquinone & $\mu \mathrm{g} / \mathrm{L}$ & 50 & $: 00$ & EPA Method 8270 \\
\hline Benzyl alcohol & $\mu \mathrm{g} / \mathrm{L}$ & 100 & 200 & EPA Method 8270 \\
\hline Beryllium & $\mu g / L$ & 0.5 & 1 & Proposed DWS (EPA, 1990) \\
\hline Beryllium-7 & $\mathrm{pCi} / \mathrm{L}$ & $3 E+03$ & $6 \mathrm{E}+03$ & Final DWS (EPA, 1977) \\
\hline Bis(2-chloroethoxy) methane & $\mu \mathrm{g} / \mathrm{L}$ & 50 & 100 & EPA Method 8270 \\
\hline Bis(2-chloroethyl) ether & $\mu \mathrm{g} / \mathrm{L}$ & 50 & 100 & EPA Method 8270 \\
\hline Bis (2-chloroisopropyl) ether & $\mu \mathrm{g} / \mathrm{L}$ & 50 & 100 & EPA Method 8270 \\
\hline Bis(chloromethyl) ether & $\mu \mathrm{g} / \mathrm{L}$ & 50 & 100 & EPA Method 8270 \\
\hline Bis(chloromethyl-ethyl) ether & $\mu \mathrm{g} / \mathrm{L}$ & 50 & 100 & EPA Method 8270 \\
\hline Bis (2-ethylhexyl) phthalate & & No flag & No flag & Set by EPD/EMS \\
\hline Bromide & $\mu g / L$ & 5,000 & 10,000 & EPA Method 300.0 \\
\hline Bromodichloromethane & $\mu \mathrm{g} / \mathrm{L}$ & 50 & 100 & Final DWS (CFR, 1991a) \\
\hline Bromoform & $\mu \mathrm{g} / \mathrm{L}$ & 50 & 100 & Final DWS (CFR, 1991a) \\
\hline Bromomethane (Methyl bromide) & $\mu g / L$ & 5 & 10 & EPA Method 8240 \\
\hline 4-Bromophenyl phenyl ether & $\mu \mathrm{g} / \mathrm{L}$ & 50 & 100 & EPA Method 8270 \\
\hline 2-sec-Butyl-4,6-dinitrophenol & $\mu \mathrm{g} / \mathrm{L}$ & 3.5 & 7 & Proposed DWS (EPA, 1990) \\
\hline Butylbenzyl phthalate & & No flag & No flag & Set by EPD/EMS \\
\hline Cadmium & $\mu \mathrm{g} / \mathrm{L}$ & 2.5 & 5 & Final DWS (CFR, 1991a) \\
\hline Calcium & & No flag & No flag & Set by EPD/EMS \\
\hline Carbon disulfide & $\mu g / L$ & 5 & 10 & EPA Method 8240 \\
\hline Carbon tetrachloride & $\mu \mathrm{g} / \mathrm{L}$ & 2.5 & 5 & Final DWS (CFR, 1991a) \\
\hline Carbon-14 & $\mathrm{pCi} / \mathrm{L}$ & $1 E+03$ & $2 E+03$ & Final DWS (EPA, 1977) \\
\hline Carbonate & $\mu g / L$ & 500 & 1,000 & EPA Method 310.1 \\
\hline Cerium-141 & $\mathrm{pCi} / \mathrm{L}$ & $1.5 E+02$ & $3 E+02$ & Final DWS (EPA, 1977) \\
\hline Cerium-144 & $\mathrm{pCi} / \mathrm{L}$ & $1.31 E+02$ & $2.61 E+02$ & Proposed DWS (EPA, 1991) \\
\hline Cesium-134 & $\mathrm{pCi} / \mathrm{L}$ & $4.07 E+01$ & $8.13 E+01$ & Proposed DWS (EPA, 1991) \\
\hline Cesium-137 & $\mathrm{pCi} / \mathrm{L}$ & $1 E+02$ & $2 E+02$ & Final DWS (EPA, 1977) \\
\hline Chlordane & $\mu g / L$ & 1 & 2 & Final DWS (CFR, 1991a) \\
\hline Chloride & $\mu \mathrm{g} / \mathrm{L}$ & 125,000 & 250,000 & Secondary DWS (CFR, 1991b) \\
\hline 4-Chloroaniline & $\mu \mathrm{g} / \mathrm{L}$ & 50 & 100 & EPA Method 8270 \\
\hline Chlorobenzene & $\mu g / L$ & 5 & 10 & EPA Method 8240 \\
\hline Chlorobenzilate & $\mu \mathrm{g} / \mathrm{L}$ & 50 & 100 & EPA Method 8270 \\
\hline Chloroethane & $\mu \mathrm{g} / \mathrm{L}$ & 5 & 10 & EPA Method 8240 \\
\hline Chloroethene (Vinyl chloride) & $\mu \mathrm{g} / \mathrm{L}$ & 1 & 2 & Final DWS (CFR, 1991a) \\
\hline Chloroethyl vinyl ether & $\mu g / L$ & 5 & 10 & EPA Method 8240 \\
\hline 2-Chloroethyl vinyl ether & $\mu \mathrm{g} / \mathrm{L}$ & 5 & 10 & EPA Method 8240 \\
\hline Chloroform & $\mu g / L$ & 50 & 100 & Final DWS (CFR, 1991a) \\
\hline para-Chloro-meta-cresol & $\mu g / L$ & 50 & 100 & EPA Method 8270 \\
\hline Chloromethane (Methyl chloride) & $\mu \mathrm{g} / \mathrm{L}$ & 5 & 10 & EPA Method 8240 \\
\hline 2-Chloronaphthalene & $\mu g / L$ & 50 & 100 & EPA Method 8240 \\
\hline
\end{tabular}




\begin{tabular}{|c|c|c|c|c|}
\hline Analyte & Unit & Flag 1 & Flag 2 & Source \\
\hline 2-Chlorophenol & $\mu \mathrm{g} / \mathrm{L}$ & 50 & 100 & EPA Method 8270 \\
\hline 4-Chlorophenyl phenyl ether & $\mu g / L$ & 50 & 100 & EPA Method 8270 \\
\hline Chloroprene & $\mu g / L$ & 1,000 & 2,000 & EPA Method 8240 \\
\hline Chromium & $\mu \mathrm{g} / \mathrm{L}$ & 50 & 100 & Final DWS (CFR, 1991a) \\
\hline Chromium-51 & $\mathrm{pCi} / \mathrm{L}$ & $3 E+03$ & $6 E+03$ & Final DWS (EPA, 1977) \\
\hline Chrysene & $\mu g / L$ & 0.1 & 0.2 & Proposed DWS (EPA, 1990) \\
\hline Cobalt & $\mu \mathrm{g} / \mathrm{L}$ & 20 & 40 & EPA Method 6010 \\
\hline Cobalt -57 & $\mathrm{pCi} / \mathrm{L}$ & $5 E+02$ & $1 E+03$ & Final DWS (EPA, 1977) \\
\hline Cobalt-58 & $\mathrm{pCi} / \mathrm{L}$ & $4.5 E+03$ & $9 E+03$ & Final DWS (EPA, 1977) \\
\hline Cobalt-60 & pCi/L & $5 E+01$ & $1 E+02$ & Final DWS (EPA, 1977) \\
\hline Color & & No flag & No flag & Set by EPD/EMS \\
\hline $\begin{array}{l}\text { Copper } \\
\text { Corrosivity }\end{array}$ & $\mu \mathrm{g} / \mathrm{L}$ & 650 & 1,300 & Final DWS (CFR, 1991a) \\
\hline m-Cresol (3-Methylphenol) & $\mu g / L$ & 50 & 100 & EPA Method 8270 \\
\hline o-Cresol (2-Methylphenol) & $\mu g / L$ & 50 & 100 & EPA Method 8270 \\
\hline p-Cresol (4-Methylphenol) & $\mu \mathrm{g} / \mathrm{L}$ & 50 & 100 & EPA Method 8270 \\
\hline Curium-242 & $\rho \mathrm{Ci} / \mathrm{L}$ & $6.65 E+01$ & $1.33 E+02$ & Proposed DWS (EPA, 1991) \\
\hline Curium-243 & $\mathrm{pCi} / \mathrm{L}$ & $4.15 E+00$ & $8.3 E+00$ & Proposed DWS (EPA, 1991) \\
\hline Curium-244 & $\mathrm{pCi} / \mathrm{L}$ & $4.92 E+00$ & $9.84 E+00$ & Proposed DWS (EPA, 1991) \\
\hline Curium-246 & $\mathrm{pCi} / \mathrm{L}$ & $3.14 E+00$ & $6.27 E+00$ & Proposed DWS (EPA, 1991) \\
\hline Cyanide & $\mu \mathrm{g} / \mathrm{L}$ & 100 & 200 & Proposed DWS (EPA, 1990 ) \\
\hline$p, p^{\prime}-D D D$ & $\mu \mathrm{g} / \mathrm{L}$ & 2.5 & 5 & EPA Method 8080 \\
\hline$p, p^{\prime}-D D E$ & $\mu \mathrm{g} / \mathrm{L}$ & 2.5 & 5 & EPA Method 8080 \\
\hline$p, p^{\prime}-D D T$ & $\mu \mathrm{g} / \mathrm{L}$ & 2.5 & 5 & EPA Method 8080 \\
\hline Di-n-butyl phthalate & & No flag & No flag & Set by EPD/EMS \\
\hline Di-n-octyl phthalate & & No flag & No flag & Set by EPD/EMS \\
\hline Diallate & $\mu \mathrm{g} / \mathrm{L}$ & 50 & 100 & EPA Method 8270 \\
\hline Dibenz $[a, h]$ anthracene & $\mu \mathrm{g} / \mathrm{L}$ & 0.15 & 0.3 & Proposed DWS (EPA, 1990) \\
\hline Dibenzofuran & $\mu \mathrm{g} / \mathrm{L}$ & 50 & 100 & EPA Method 8270 \\
\hline Dibromochloromethane & $\mu \mathrm{g} / \mathrm{L}$ & 50 & 100 & Final DWS (CFR, 1991a) \\
\hline Dibromochloropropane & $\mu \mathrm{g} / \mathrm{L}$ & 0.1 & 0.2 & Final DWS (CFR, 1991a) \\
\hline 1,2-Dibromo-3-chloropropane & $\mu \mathrm{g} / \mathrm{L}$ & 250 & 500 & EPA Method 8240 \\
\hline 1,2-Dibromoethane & $\mu g / L$ & 100 & 200 & EPA Method 8240 \\
\hline \multicolumn{5}{|l|}{ Dibromomethane } \\
\hline (Methylene bromide) & $\mu \mathrm{g} / \mathrm{L}$ & 5 & 10 & EPA Method 8240 \\
\hline 1,2-Dichlorobenzene & $\mu \mathrm{g} / \mathrm{L}$ & 300 & 600 & Final DWS (CFR, 1991a) \\
\hline 1,3-Dichlorobenzene & $\mu \mathrm{g} / \mathrm{L}$ & 50 & 100 & EPA Method 8270 \\
\hline 1,4-Dichlorobenzene & $\mu \mathrm{g} / \mathrm{L}$ & 37.5 & 75 & Final DWS (CFR, 1991a) \\
\hline 3,3'-Dichlorobenzidine & $\mu \mathrm{g} / \mathrm{L}$ & 50 & 100 & EPA Method 8270 \\
\hline trans-1,4-Dichloro-2-butene & $\mu \mathrm{g} / \mathrm{L}$ & 150 & 300 & EPA Method 8240 \\
\hline Dichlorodifluoromethane & $\mu g / L$ & 5 & 10 & EPA Method 8240 \\
\hline 1,1-Dichloroethane & $\mu \mathrm{g} / \mathrm{L}$ & 5 & 10 & EPA Method 8240 \\
\hline 1,2-Dichloroethane & $\mu g / L$ & 2.5 & 5 & Final DWS (CFR, 1991a) \\
\hline cis-1,2-Dichloroethene & $\mu g / L$ & 35 & 70 & Final DWS (CFR, 1991a) \\
\hline 1,1-Dichloroethylene & $\mu \mathrm{g} / \mathrm{L}$ & 3.5 & 7 & Final DWS (CFR, 1991a) \\
\hline 1,2-Dichloroethylene & $\mu \mathrm{g} / \mathrm{L}$ & 25 & 50 & EPA Method 8240 \\
\hline trans-1,2-Dichloroethylene & $\mu g / L$ & 50 & 100 & Final DWS (CFR, 1991a) \\
\hline \multicolumn{5}{|l|}{ Dichloromethane } \\
\hline (Methylene cinloride) & & No flag & No flag & Set by EPD/EMS \\
\hline 2,4-Dichlorophenol & $\mu \mathrm{g} / \mathrm{L}$ & 50 & 100 & EPA Method 8270 \\
\hline 2,6-Dichlorophenol & $\mu g / L$ & 50 & 100 & EPA Method 8270 \\
\hline 2,4-Dichlorophenoxyacetic acid & $\mu \mathrm{g} / \mathrm{L}$ & 35 & 70 & Final DWS (CFR, 1991a) \\
\hline 1,2-Dichloropropane & $\mu \mathrm{g} / \mathrm{L}$ & 2.5 & 5 & Final DWS (CFR, 1991a) \\
\hline cis-1,3-Dichloropropene & $\mu \mathrm{g} / \mathrm{L}$ & 5 & 10 & EPA Method 8240 \\
\hline trans-1,3-Dichloropropene & $\mu \mathrm{g} / \mathrm{L}$ & 5 & 10 & EPA Method 8240 \\
\hline Dieldrin & $\mu \mathrm{g} / \mathrm{L}$ & 2.5 & 5 & EPA Method 8080 \\
\hline
\end{tabular}




\begin{tabular}{|c|c|c|c|c|}
\hline Anaiyte & Unit & Flag 1 & Flag 2 & Source \\
\hline Diethyl phthalate & & No flag & No flag & Set by EPD/EMS \\
\hline Dimethoate & $\mu \mathrm{g} / \mathrm{L}$ & 50 & 100 & EPA Method 8270 \\
\hline p-Dimethylaminoazobenzene & $\mu \mathrm{g} / \mathrm{L}$ & 50 & 100 & EPA Method 8270 \\
\hline p-(Dimethylamino)ethylbenzene & $\mu g / L$ & 50 & 100 & EPA Method 8270 \\
\hline 7,12-Dimethylbenzla]anthracene & $\mu \mathrm{g} / \mathrm{L}$ & 50 & 100 & EPA Method 8270 \\
\hline 3,3'-Dimethylbenzidine & $\mu g / L$ & 50 & 100 & EPA Method 8270 \\
\hline a,a-Dimethylphenethylamine & $\mu g / L$ & 50 & 100 & EPA Method 8270 \\
\hline 2,4-Dimethyl phenol & $\mu \mathrm{g} / \mathrm{L}$ & 50 & 100 & EPA Method 8270 \\
\hline Dimethyl phthalate & & No flag & No flag & Set by EPD/EMS \\
\hline 1,3-Dinitrobenzene & $\mu g / L$ & 50 & 100 & EPA Method 8270 \\
\hline 4,6-Dinitro-ortho-cresol & $\mu \mathrm{g} / \mathrm{L}$ & 250 & 500 & EPA Method 8270 \\
\hline 2,4-Dinitrophenol & $\mu g / L$ & 250 & 500 & EPA Method 8270 \\
\hline 2,4-Dinitrotoluene & $\mu g / L$ & 50 & 100 & EPA Method 8270 \\
\hline 2,6-Dinitrotoluene & $\mu g / L$ & 50 & 100 & EPA Method 8270 \\
\hline 1,4-Dioxane & $\mu \mathrm{g} / \mathrm{L}$ & 50 & 100 & EPA Method 8270 \\
\hline Diphenylamine & $\mu \mathrm{g} / \mathrm{L}$ & 50 & 100 & EPA Method 8270 \\
\hline 1,2-Diphenylhydrazine & $\mu \mathrm{g} / \mathrm{L}$ & 50 & 100 & EPA Method 8270 \\
\hline Dissolved organic carbon & $\mu \mathrm{g} / \mathrm{L}$ & 5,000 & 10,000 & EPA Method 9060 \\
\hline Disulfoton & $\mu g / L$ & 50 & 100 & EPA Method 8270 \\
\hline Eh & & No flag & No flag & Set by EPD/EMS \\
\hline alpha-Endosulfan & $\mu g / L$ & 50 & 100 & EPA Method 8270 \\
\hline beta-Endosulfan & $\mu \mathrm{g} / \mathrm{L}$ & 50 & 100 & EPA Method 8270 \\
\hline Endosulfan 1 & $\mu g / L$ & 2.5 & 5 & EPA Method 8080 \\
\hline Endosulfan II & $\mu g / L$ & 2.5 & 5 & EPA Method 8080 \\
\hline Endosulfan sulfate & $\mu \mathrm{g} / \mathrm{L}$ & 2.5 & 5 & EPA Method 8080 \\
\hline Endrin & $\mu \mathrm{g} / \mathrm{L}$ & 0.1 & 0.2 & Final DWS (CFR, 1991a) \\
\hline Endrin aldehyde & $\mu g / L$ & 2.5 & 5 & EPA Method 8080 \\
\hline Endrin ketone & & No flag & No flag & Set by EPD/EMS \\
\hline Ethyl methacrylate & $\mu \mathrm{g} / \mathrm{L}$ & 50 & 100 & EPA Method 8270 \\
\hline Ethyl methanesulfonate & $\mu g / L$ & 50 & 100 & EPA Method 8270 \\
\hline Ethylbenzene & $\mu \mathrm{g} / \mathrm{L}$ & 350 & 700 & Final DWS (CFR, 1991a) \\
\hline Europium-154 & $\mathrm{pCi} / \mathrm{L}$ & $1 E+02$ & $2 E+02$ & Final DWS (EPA, 1977) \\
\hline Europium-155 & $\mathrm{pCi} / \mathrm{L}$ & $3 E+02$ & $6 E+02$ & Final DWS (EPA, 1977) \\
\hline Famphur & $\mu \mathrm{g} / \mathrm{L}$ & 50 & 100 & EPA Method 8270 \\
\hline Fluoranthene & $\mu g / L$ & 50 & 100 & EPA Method 8270 \\
\hline Fluorene & $\mu g / L$ & 50 & 100 & EPA Method 8270 \\
\hline Fluoride & $\mu \mathrm{g} / \mathrm{L}$ & 2,000 & 4,000 & Final DWS (CFR, 1991a) \\
\hline Gross alpha & $\mathrm{pCi} / \mathrm{L}$ & $7.5 E+00$ & $1.5 E+01$ & Final DWS (CFR, 1991a) \\
\hline Heptachlor & $\mu g / L$ & 0.2 & 0.4 & Final DWS (CFR, 1991a) \\
\hline Heptachlor epoxide & $\mu \mathrm{g} / \mathrm{L}$ & 0.1 & 0.2 & Final DWS (CFR, 1991a) \\
\hline $\begin{array}{l}\text { Heptachlorodibenzo-p-dioxin } \\
\text { isomers }\end{array}$ & $\mu \mathrm{g} / \mathrm{L}$ & 0.00325 & 0.0065 & EPA Method 8280 \\
\hline $\begin{array}{l}\text { 1,2,3,4,6,7,8-Heptachlorodibenzo- } \\
\text { p-dioxin }\end{array}$ & $\mu \mathrm{g} / \mathrm{L}$ & 0.00325 & 0.0065 & EPA Method 8280 \\
\hline Heptachlorodibenzo-p-furan & & & & \\
\hline $\begin{array}{l}\text { isomers } \\
1,2,3,4,6,7,8-H e p t a c h l o r o d i b e n z o-\end{array}$ & $\mu \mathrm{g} / \mathrm{L}$ & 0.00225 & 0.0045 & EPA Method 8280 \\
\hline$p$-furan & $\mu \mathrm{g} / \mathrm{L}$ & 0.00225 & 0.0045 & EPA Method 8280 \\
\hline Hexachlorobenzene & $\mu g / L$ & 0.5 & 1 & Proposed DWS (EPA, 1990) \\
\hline Hexachlorobutadiene & $\mu \mathrm{g} / \mathrm{L}$ & 50 & 100 & EPA Method 8270 \\
\hline Hexachlorocyclopentadiene & $\mu \mathrm{g} / \mathrm{L}$ & 25 & 50 & Proposed DWS (EPA, 1990) \\
\hline $\begin{array}{l}\text { Hexachlorodibenzo-p-dioxin isomers } \\
1,2,3,4,7,8 \text {-Hexachlorodibenzo- }\end{array}$ & $\mu g / L$ & 0.00225 & 0.0045 & EPA Method 8280 \\
\hline p-dioxin & $\mu g / L$. & 0.00225 & 0.0045 & EPA Method 8280 \\
\hline Hexachlorodibenzo-p-furan isomers & $\mu g / L$ & 0.002 & 0.004 & EPA Method 8280 \\
\hline
\end{tabular}


Analvte

$1,2,3,4,7,8$-Hexachlorodibenzop-furan

Hexachloroethane

Hexachlorophene

Hexachloropropene

2-Hexanone

Indeno[1,2,3-c, O]pyrene

lodine

lodine-129

lodine-131

lodomethane (Methyl iodide)

Iron

Iron-55

iron-59

Isobutyl aicohol

Isrjuin

Isophorone

Isosafrole

Kepone

Lanthanum-140

Lead

Lindane

Lithium

Magnesium

Manganese

Manganese-54

Mercury

Methacrylonitrile

Methapyrilene

Methoxychlor

3-Methylcholanthrene

2-Methyl-4,6-dinitrophenol

Methyl ethyl ketone

Methyl isobutyl ketone

Methyl methacrylate

Methyl methanesulfonate

2-Methy/naphthalene

Molybdenum

Naphthalene

1.4-Naphthoquinone

1-Naphthylamine

2-Naphthylarrine

Neptunium-237

Nickel

Nickel-59

Nickel-63

Niobium-95

Nitrate as nitrogen

Nitrite as nitrogen

2-Nitroaniline

3-Nitroaniline

4-Nitroaniline

Nitrobenzene

Nitrogen by Kjeldahl method

2-Nitrophenol

4-Nitrophanol

\begin{tabular}{|c|c|c|c|}
\hline Unit & Flag 1 & Flag 2 & Source \\
\hline$\mu g / L$ & 0.002 & 0.004 & EPA Method 8280 \\
\hline$\mu g / L$ & 50 & 100 & EF'A Method 8270 \\
\hline$\mu g / L$ & 250 & 500 & EFA Method 8270 \\
\hline$\mu g / L$ & 50 & 100 & EFA Method 8270 \\
\hline$\mu g / h$ & 100 & 200 & EFA Method 8240 \\
\hline$\mu g / L$ & 50 & 100 & EPA Method 8270 \\
\hline$\mu g / L$ & 500 & 1,000 & EPA Method 415 \\
\hline $\mathrm{pCi} / \mathrm{L}$ & 5E-01 & $1 E+00$ & Final DWS (EFA, 1977) \\
\hline $\mathrm{pCi} / \mathrm{L}$ & $1.5 E+00$ & $3 E+00$ & Final DWS (EPA, 1977) \\
\hline$\mu g / L$ & 75 & 150 & EFA Method 8240 \\
\hline$\mu g / L$ & 150 & 300 & Secondary DWS (CFR, 1991b) \\
\hline $\mathrm{pCi} / \mathrm{L}$ & $1 E+03$ & $2 E+03$ & Final DWS (EPA, 1977) \\
\hline $\mathrm{pCi} / \mathrm{L}$ & $1 E+02$ & $2 E+02$ & Final DWS (EPA, 1977) \\
\hline$\mu g / L$ & 500 & 1,000 & EPA Method 8240 \\
\hline$\mu g / L$ & 50 & 100 & EPA Method $82 ; 0$ \\
\hline$\mu g / L$ & 50 & 100 & EPA Method 8270 \\
\hline$\mu g / L$ & 50 & 100 & EPA Method 8270 \\
\hline$\mu g / L$ & 50 & 100 & EPA Method 8270 \\
\hline $\mathrm{pCi} / \mathrm{L}$ & $3 E+01$ & $6 E+01$ & Final DWS (EPA, 1977) \\
\hline$\mu g / L$ & 7.5 & 15 & Final DWS (CFR, 1991a) \\
\hline$\mu g / L$ & 0.1 & 0.2 & Final DWS (CFR, 1991a) \\
\hline$\mu g / L$ & 25 & 50 & EPA Method 6010 \\
\hline & No flag & No flag & Set by EPD/EMS \\
\hline$\mu g / L$ & 25 & 50 & Secondary DWS (CFR, 1991b) \\
\hline $\mathrm{pCi} / \mathrm{L}$ & $1.5 E+02$ & $3 E+02$ & Final DWS (EPA, 1977) \\
\hline$\mu g / L$ & 1 & 2 & Final DWS (CFR, 1991a) \\
\hline$\mu g / L$ & 250 & 500 & EPA Method 8240 \\
\hline$\mu g / L$ & 50 & 100 & EPA Method 8270 \\
\hline$\mu g / L$ & 20 & 40 & Final DWS (CFR, 1991a) \\
\hline$\mu g / L$ & 50 & 100 & EPA Method 8270 \\
\hline$\mu g / L$ & 250 & 500 & EPA Method 8270 \\
\hline & No flag & No flag & Set by EPD/EMS \\
\hline & No flag & No flag & Set by EPD/EMS \\
\hline$\mu g / L$ & 50 & 100 & EPA Method 8270 \\
\hline$\mu g / L$ & 50 & 100 & EPA Method 8270 \\
\hline$\mu g / L$ & 50 & 100 & EPA Method 8270 \\
\hline$\mu g / L$ & 250 & 500 & EPA Method 6010 \\
\hline$\mu g / L$ & 50 & 100 & EPA Method 8270 \\
\hline$\mu g / L$ & 50 & 100 & EPA Method 8270 \\
\hline$\mu g / L$ & 50 & 100 & EPA Method 8270 \\
\hline$\mu g / L$ & 50 & 100 & EPA Method 8270 \\
\hline $\mathrm{pCi} / \mathrm{L}$ & $3.53 E+00$ & $7.06 E+00$ & Proposed DWS (EPA, 1991) \\
\hline$\mu g / L$ & 50 & 100 & Proposed DWS (EPA, 1990) \\
\hline $\mathrm{pCi} / \mathrm{L}$ & $1.5 E+02$ & $3 E+02$ & Final DWS (EPA, 1977) \\
\hline pCi/L & $2.5 E+01$ & $5 E+01$ & Final DWS (EPA, 1977) \\
\hline $\mathrm{pCi} / \mathrm{L}$ & $1.5 E+02$ & $3 E+02$ & Final DWS (EPA, 1977) \\
\hline$\mu g / L$ & 5,000 & 10,000 & Final DWS (CFR, 1991a) \\
\hline$\mu g / L$ & 500 & 1,000 & Final DWS (CFR, 1991a) \\
\hline$\mu g / L$ & 50 & 100 & EPA Method 8270 \\
\hline$\mu g / L$ & 50 & 100 & EPA Method 8270 \\
\hline$\mu g / L$ & 50 & 100 & EPA Method 8270 \\
\hline$\mu g / L$ & 50 & 100 & EPA Method 8270 \\
\hline$\mu g / L$ & 500 & 1,000 & EPA Method 351.2 \\
\hline$\mu g / L$ & 50 & 100 & EPA Method 8270 \\
\hline$\mu g / L$ & 50 & 100 & EPA Method 8270 \\
\hline
\end{tabular}




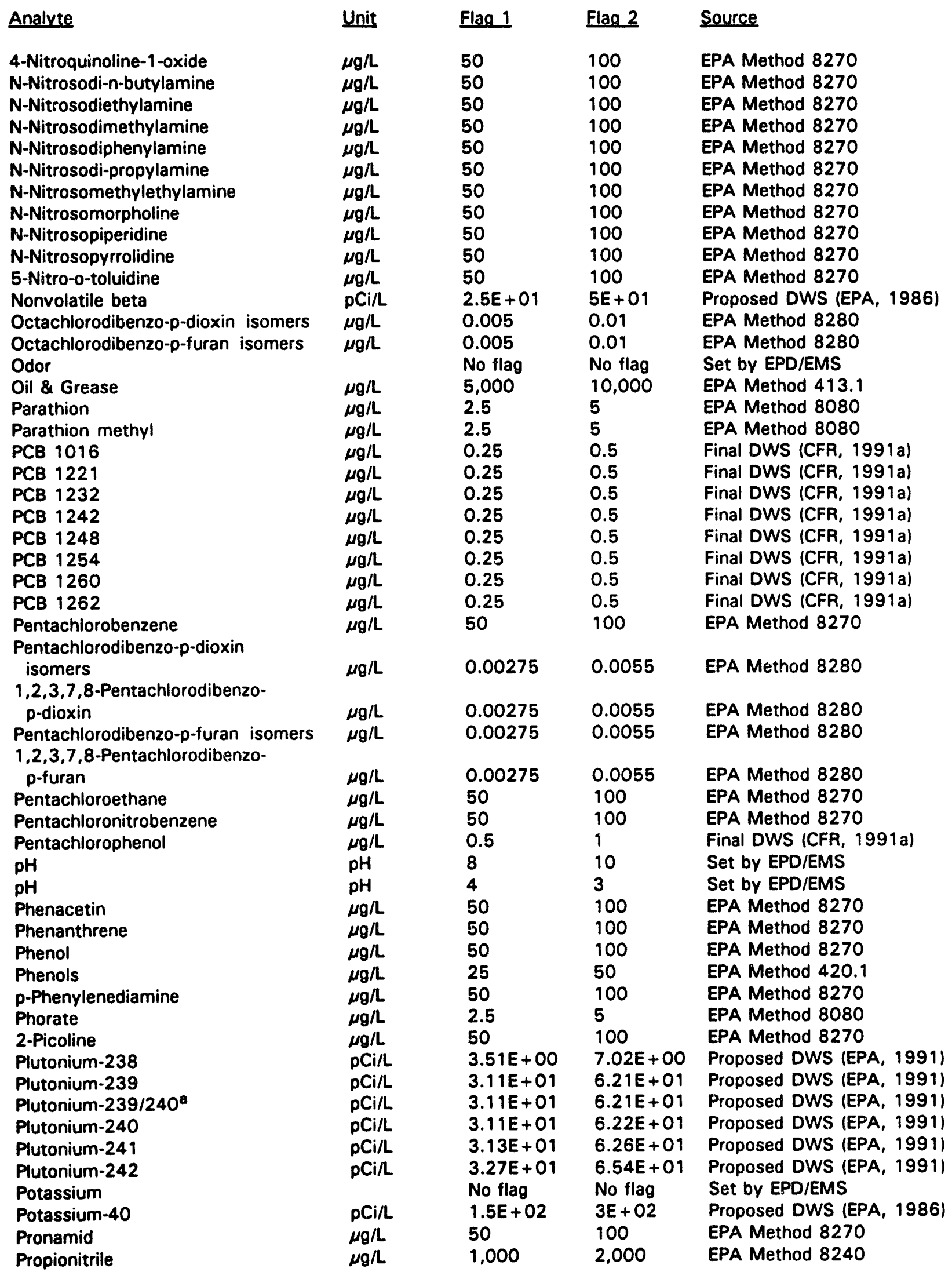


Analvte

Pyrene

Pyridine

Radium-226

Radium-228

Radon-222

Ruthenium-103

Ruthenium-106

Safrole

Selenium

Silica

Silver

Sodium

Sodium-22

Specific conductance

Strontium-89

Strontium-89/90

Strontium-90

Styrene

Sulfate

Sulfide

Sulfotepp

Surfactants

2,3,7,8-TCDD

$2,3,7,8-T C D F$

Technetium-99

1,2,4,5-Tetrachlorobenzene

Tetrachlorodibenzo-p-dioxin isomers

Tetrachlorodibenzo-p-furan isomers

1,1,1,2-Tetrachloroethane

1,1,2,2-Tetrachloroethane

Tetrachloroethylene

2,3,4,6-Tetrachlorophenol

Tetraethyl dithiopyrophosphate

Thallium

Thionazin

Thorium-228

Thorium-230

Thorium-232

Thorium-234

Tin

Tin-113

Tolvene

o-Toluidine

Total carbon

Total dissolved solids

Total hydrocarbons

Total inorganic carbon

Total organic carbon

Total organic halogens

Total organic nitrogen

Total petroleum hydrocarbons

Total phosphates (as P)

Total phosphorus

Total radium

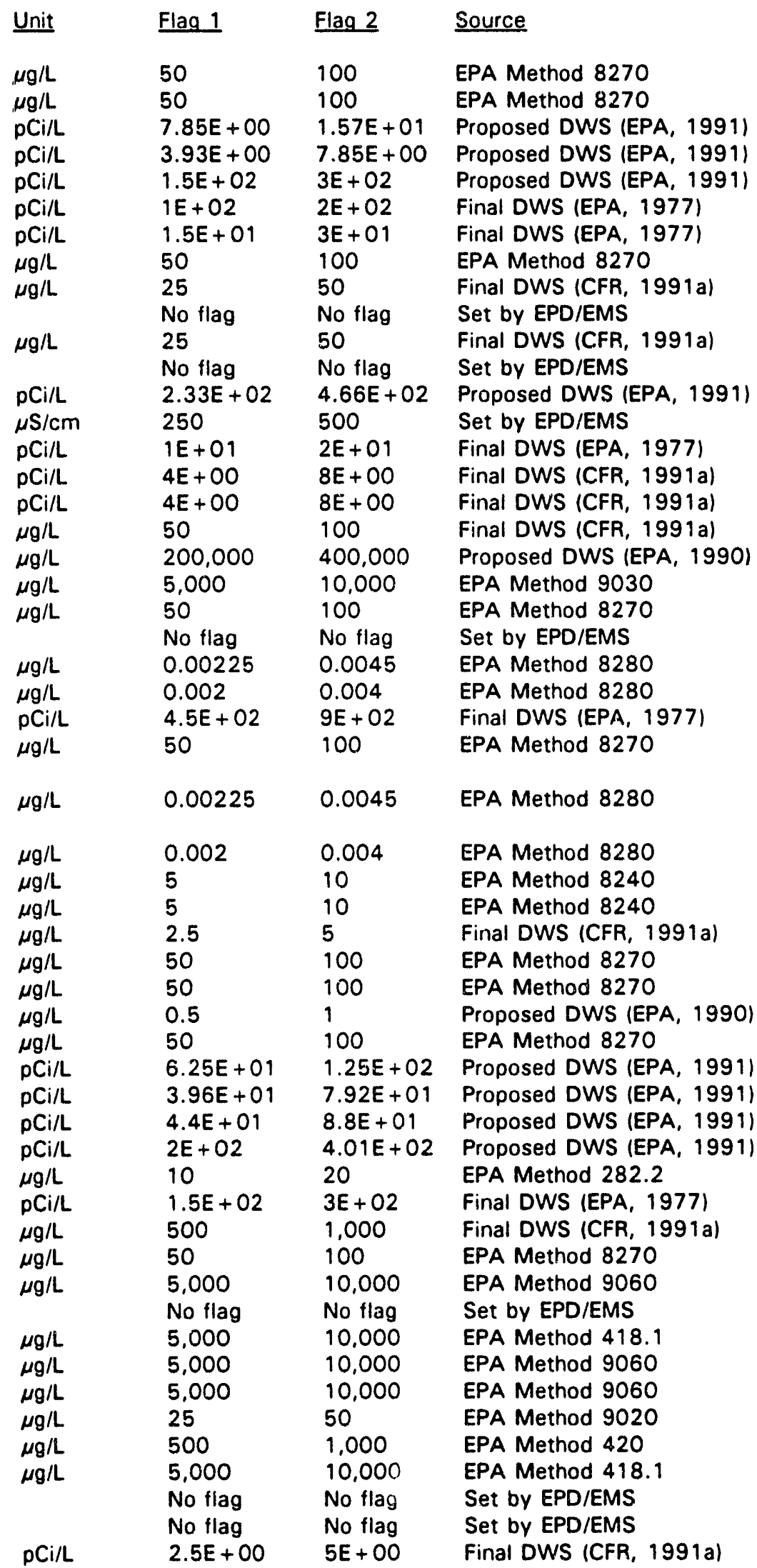




\section{Analyte}

Total silica

Total trihalomethanes

Toxaphene

2,4,5-TP (Silvex)

Tributyl phosphate

1,2,4-Trichlorobenzene

1,1,1-Trichloroethane

1,1,2-Trichloroethane

Trichloroethylene

Trichlorofluoromethane

2,4,5-Trichlorophenol

2,4,6-Trichlorophenol

2,4,5-Trichlorophenoxyacetic acid

1,2,3-Trichloropropane

$0,0,0$-Triethyl phosphorothioate

1,3,5-Trinitrobenzene

Tritium

Turbidity

Uranium

Uranium alpha activity

Uranium-233/234 ${ }^{\mathrm{a}}$

Uranium-234

Uranium-235

Uranium-238

Vanadium

Vinyl acetate

Xylenes

Zinc

Zinc-65

Zirconium-95

Zirconium/Niobium-95

\begin{tabular}{|c|c|c|}
\hline Unit & Flag 1 & Flag 2 \\
\hline$\mu g / L$ & 500 & 1,000 \\
\hline$\mu g / L$ & 50 & 100 \\
\hline$\mu g / L$ & 1.5 & 3 \\
\hline$\mu g / L$ & 25 & 50 \\
\hline$\mu g / L$ & 50 & 100 \\
\hline$\mu g / L$ & 4.5 & 9 \\
\hline$\mu \mathrm{g} / \mathrm{L}$ & 100 & 200 \\
\hline$\mu \mathrm{g} / \mathrm{L}$ & 2.5 & 5 \\
\hline$\mu \mathrm{g} / \mathrm{L}$ & 2.5 & 5 \\
\hline$\mu g / L$ & 5 & 10 \\
\hline$\mu \mathrm{g} / \mathrm{L}$ & 50 & 100 \\
\hline$\mu g / L$ & 50 & 100 \\
\hline$\mu \mathrm{g} / \mathrm{L}$ & 2.5 & 5 \\
\hline$\mu g / L$ & 5 & 10 \\
\hline$\mu g / L$ & 50 & 100 \\
\hline$\mu \mathrm{g} / \mathrm{L}$ & 50 & 100 \\
\hline \multirow[t]{2}{*}{$\mathrm{pCi} / \mathrm{mL}$} & $1 E+01$ & $2 E+01$ \\
\hline & No flag & No flag \\
\hline$\mu g / L$ & 10 & 20 \\
\hline$p \mathrm{Ci} / \mathrm{L}$ & $1.5 E+01$ & $3 E+01$ \\
\hline $\mathrm{pCi} / \mathrm{L}$ & $6.9 E-00$ & $1.38 E+01$ \\
\hline $\mathrm{pCi} / \mathrm{L}$ & $6.95 E+00$ & $1.39 E+01$ \\
\hline $\mathrm{pCi} / \mathrm{L}$ & $7.25 E+00$ & $1.45 E+01$ \\
\hline $\mathrm{oCi} / \mathrm{L}$ & $7.3 E+00$ & $1.46 \mathrm{E}+01$ \\
\hline$\mu g / L$ & 50 & 100 \\
\hline$\mu g / L$ & 5 & 10 \\
\hline$\mu g / L$ & 5,000 & 10,000 \\
\hline$\mu g / L$ & 2,500 & 5,000 \\
\hline $\mathrm{pCi} / \mathrm{L}$ & $1.5 E+02$ & $3 E+02$ \\
\hline $\mathrm{pCi} / \mathrm{L}$ & $1 E+02$ & $2 E+02$ \\
\hline $\mathrm{pCi} / \mathrm{L}$ & $1 E+02$ & $2 E+02$ \\
\hline
\end{tabular}

Source

EPA Method 6010

Final DWS (CFR, 1991a)

Final DWS (CFR, 1991a)

Final DWS (CFR, 1991a)

EPA Method 8270

Proposed DWS (EPA, 1990)

Final DWS (CFR, 1991a)

Proposed DWS (EPA, 1990 )

Final DWS (CFR, 1991a)

EPA Method 8240

EPA Method 8270

EPA Method 8270

EPA Method 8150

EPA Method 8240

EPA Method 8270

EPA Method 8270

Final DWS (CFR, 1991a)

Set by EPD/EMS

Proposed DWS (EPA, 1991)

Proposed C NS (EPA, 1991)

Proposed DWS (EPA, 1991)

Proposed DWS (EPA, 1991)

Proposed DWS (EPA, 1991)

Proposed DWS (EPA, 1991)

EPA Method 6010

EPA Method 8240

Final DWS (CFR, 1991a)

Secondary DWS (CFR, 1991b)

Final DWS (EPA, 1977)

Final DWS (EPA, 1977)

Final DWS (EPA, 1977)

a For double radionuclide analyses where each separate radionuclide has its own standard, the more stringent standard is used.

\section{References}

APHA (American Public Health Association, American Water Works Association, and Water Pollution Control Federation), 1985. Standard Methods for the Examination of Water and Wastewates 16th edition. Washington, DC.

CFR (Code of Federal Regulations), 1991a. National Primary Drinking Water Regulations, 40 CFR, Part 141, pp. 578-715. Washington, DC.

CFR (Code of Federal Regulations), 1991b. National Secondary Drinking Water Regulations, 40 CFR, Part 143, pp. 758-762. Washington, DC.

EPA (U.S. Environmental Protection Agency), 1977. National Interim Primary Drinking Water Regulations, EPA-570/9-76-003. Washington, DC. 
EPA (U.S. Environmental Protection Agency), 1986. Water Pollution Control; National Primary Drinking Water Regulations, Radionuclides (Proposed). Federal Register, September 30, 1986, pp. 34836-34862. Washington, DC.

EPA (U.S. Environmental Protection Agency), 1990. National Primary and Secondary Drinking Water Regulations; Synthetic Organic Chemicals and Inorganic Chemicals (Proposed Rule). Federal Register, July 25, 1990, pp. 30369-30448. Washington, DC.

EPA (U.S. Environmental Protection Agency), 1991. National Primary Drinking Water Regulations; Radionuclides; Proposed Rule. Federal Register, July 18, 1991, pp. 33052-33127. Washington, DC. 
Appendix C - Figures 


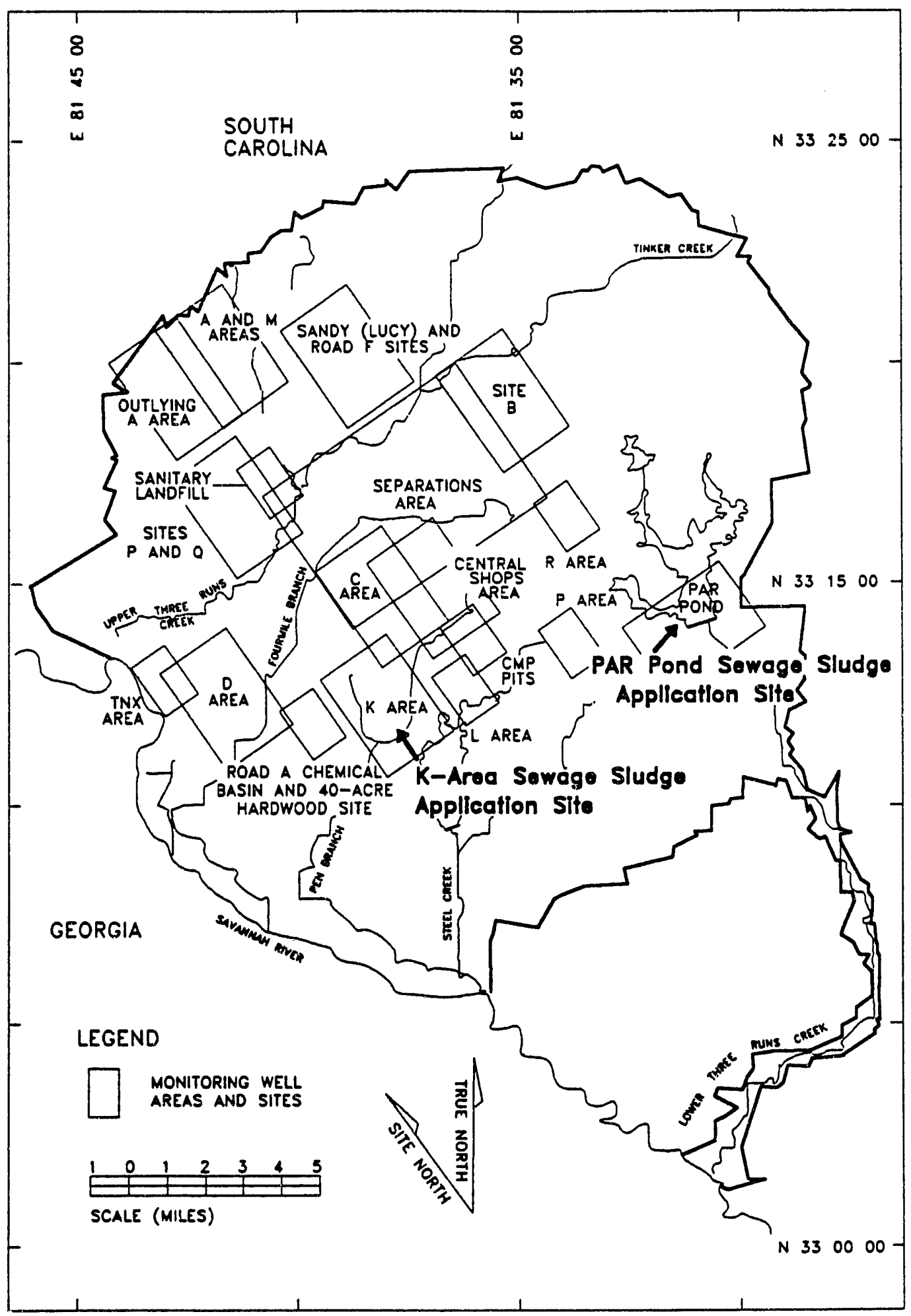

Figure 1. Location of the K-Area and Par Pond Sewage Sludge Application Sites at the Savannah River Site 


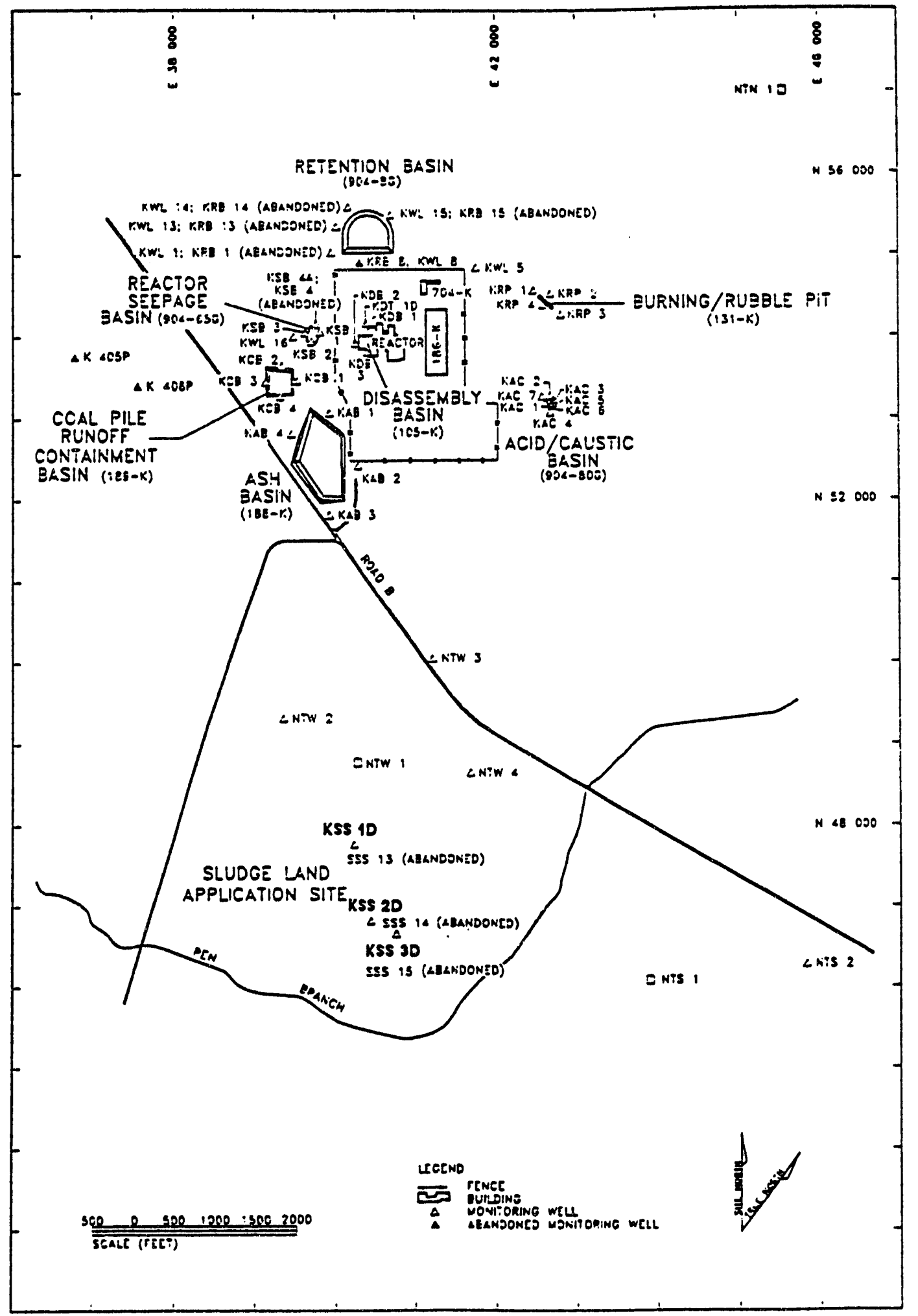

Figure 2. Location of Groundwater Monitoring Wells at the K-Area Sewage Sludge Application Site 


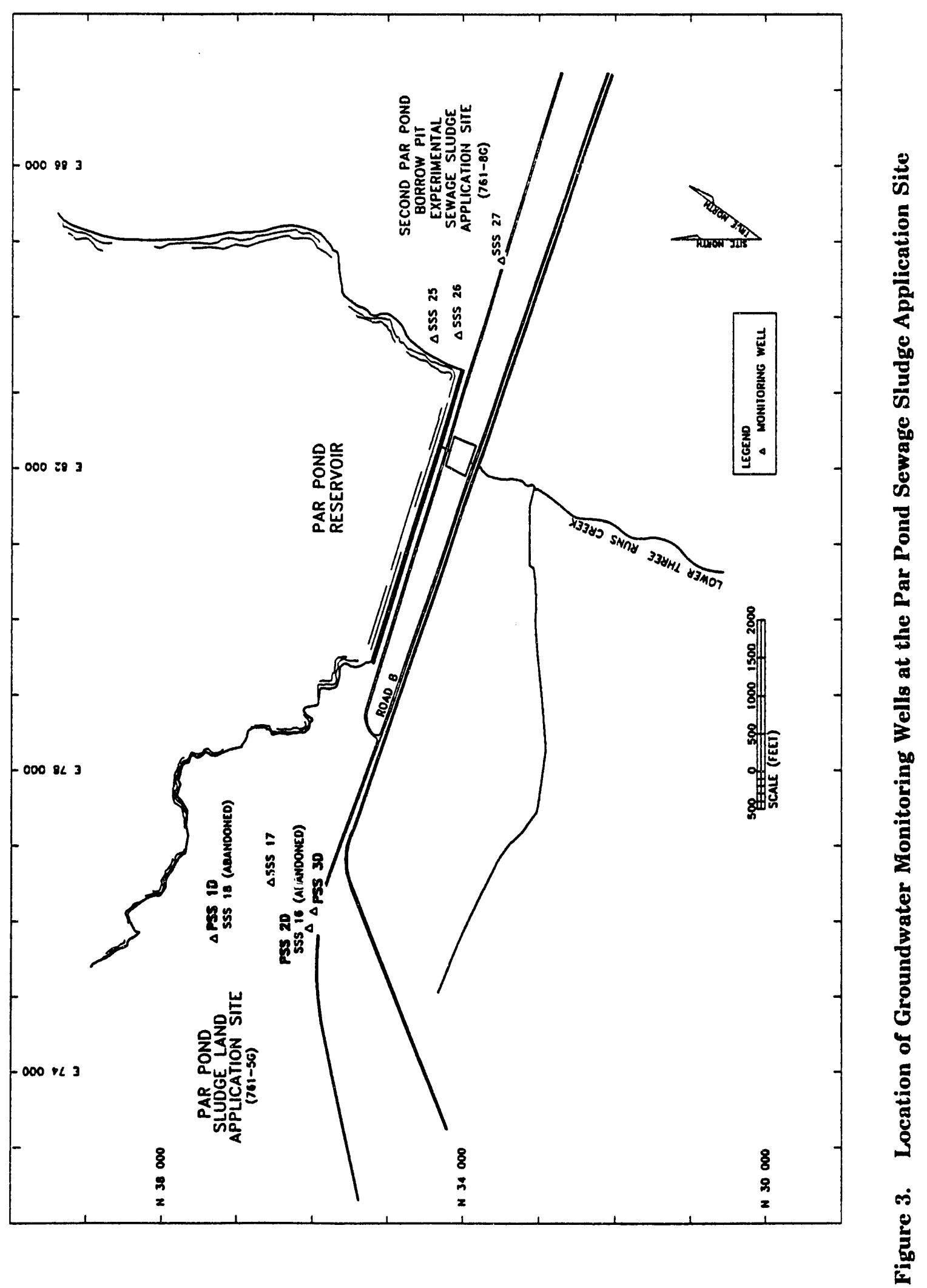




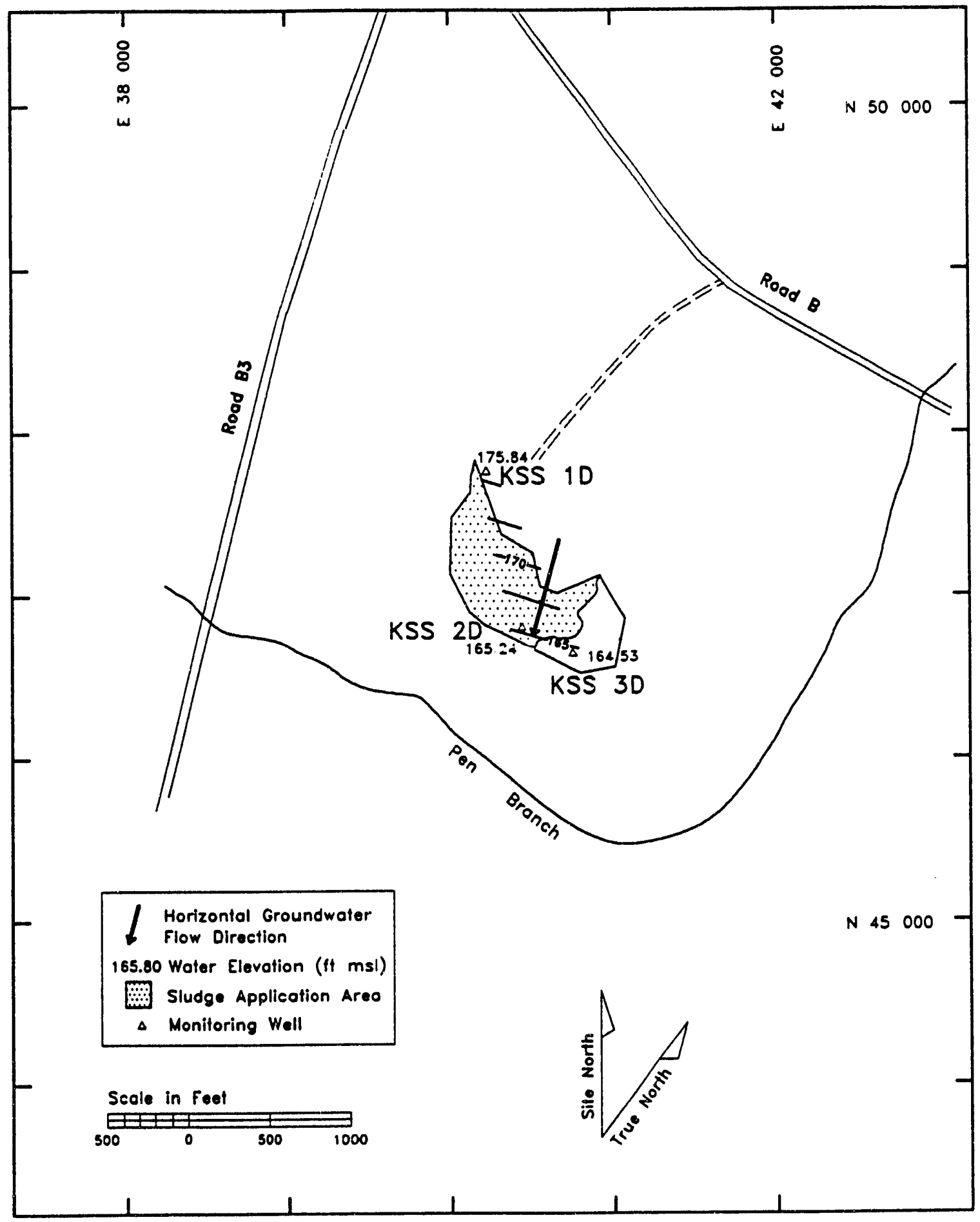

Figure 4. Potentiometric Surface Map of the K-Area Sewage Sludge Application Site 


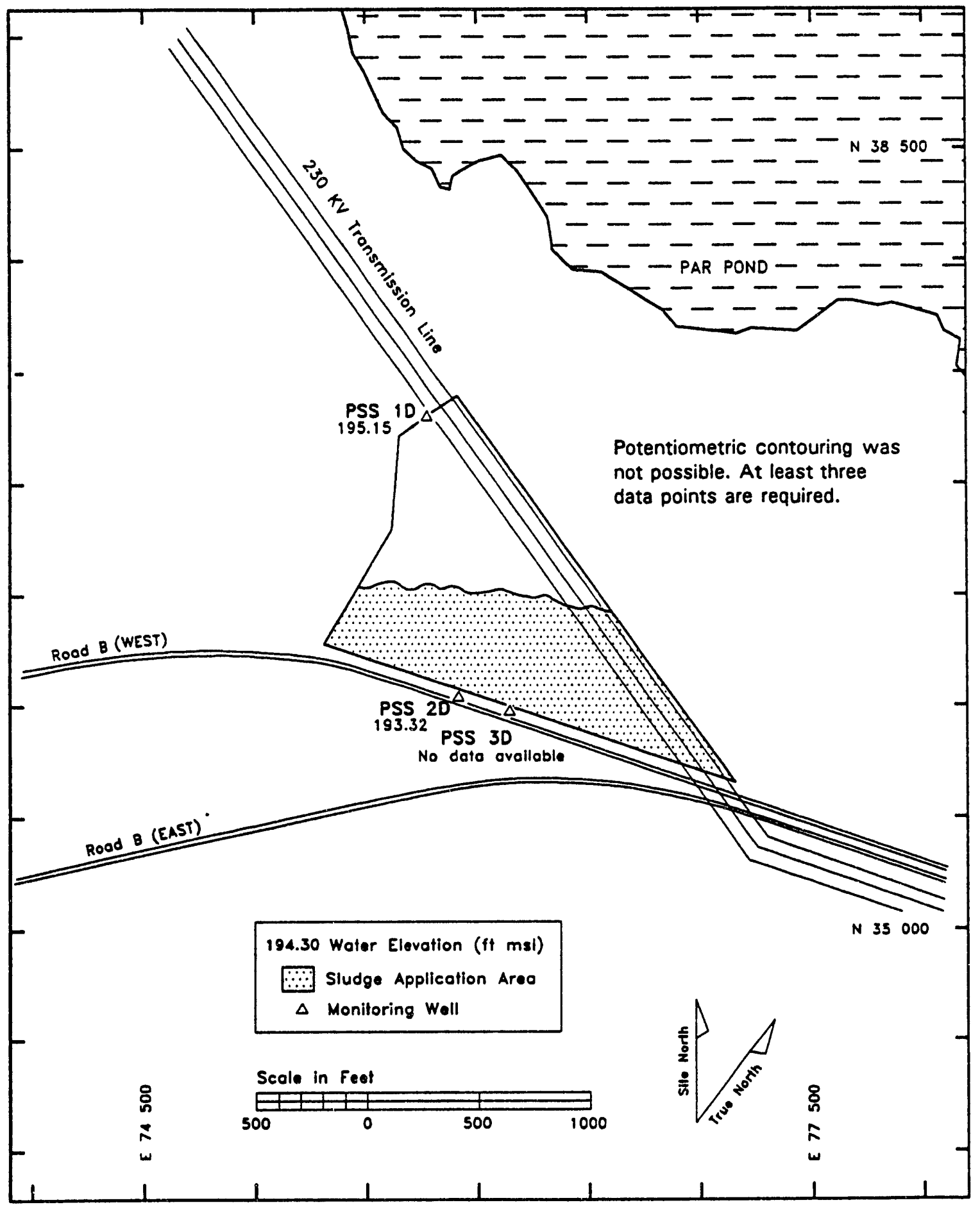

Figure 5. Potentiometric Surface Map of the Par Pond Sewage Sludge Application Site 
Appendix D - Groundwater Monitoring Results Tables 


\section{Key to Reading the Tables}

The following abbreviations may appear in the tabular data:

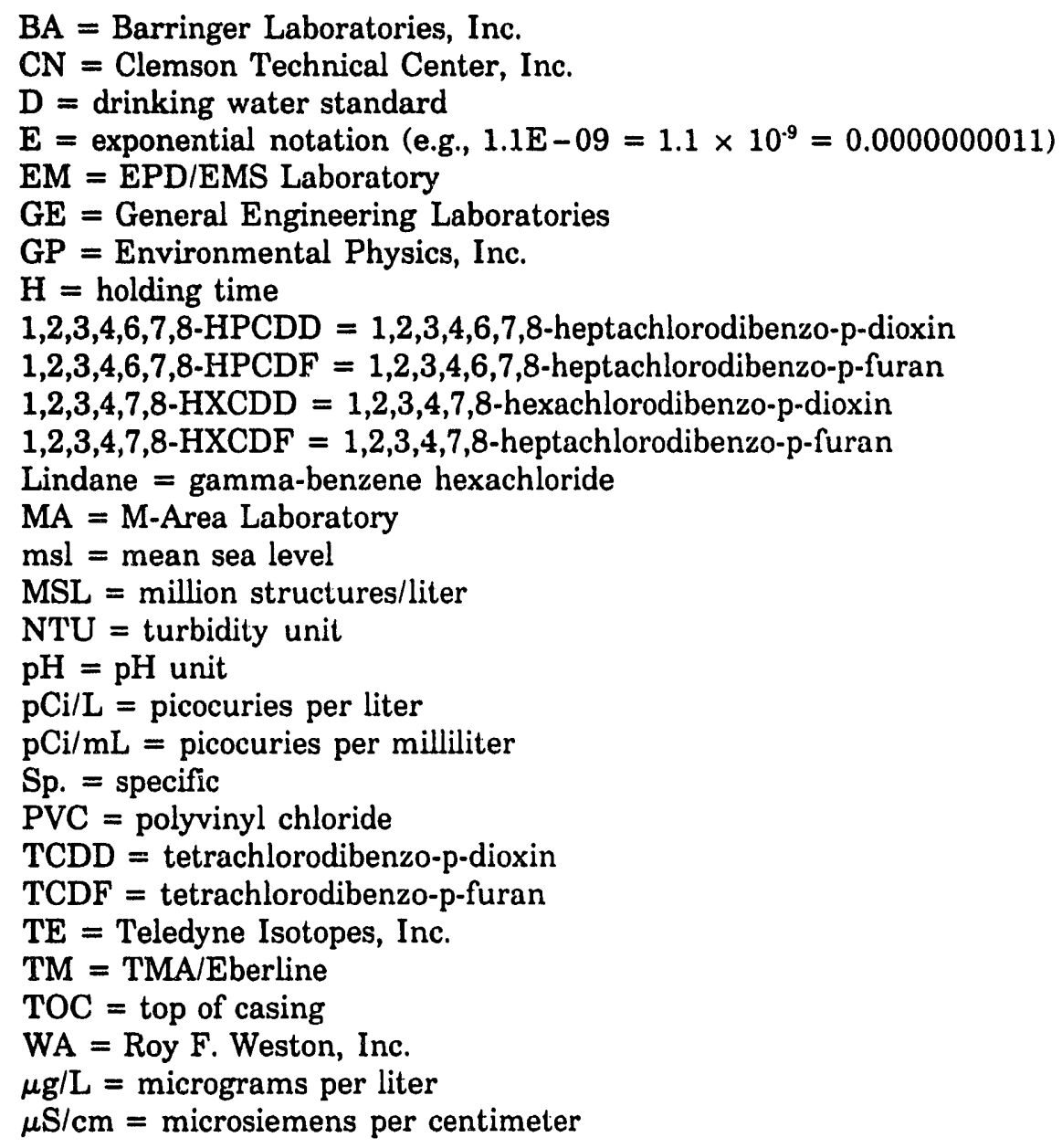

\section{Holding Times}

Standard analytical methods include a limit, called holding time, on the maximum elapsed time between sample collection and extraction or analysis by the laboratory. In the data tables, a large $\operatorname{dot}(\bullet)$ in the $\mathrm{H}$ (holding time) column indicates that holding time was exceeded. Analyses performed beyond holding time may not yield valid results.

South Carolina Department of Health and Environmental Control (SCDHEC) allows only 15 minutes to elapse between sampling and analysis for $\mathrm{pH}$. Thus, laboratory $\mathrm{pH}$ analyses always exceed holding time.

Laboratory-initiated procedures for reducing the number of other analyses performed out of holding time include subcontracting analyses when difficulties with equipment, personnel, or work load would prevent timely analyses. Beginning fourth quarter 1991, SRS reduced the compensation to laboratories for analyses performed out of holding time. 
Table 1. Constituents Exceeding the Primary Drinking Water Standards at the K-Area Sewage Sludge Application Site

$\begin{array}{lllll}\text { Well } & \text { Constituent } & \underline{\text { Unit }} & \underline{1092} & \underline{2092} \\ \text { KSS 2D } & \text { Lead } & \mu \mathrm{g} / \mathrm{L} & 16^{\mathrm{a}} & \text { NA } \\ & \text { Lead } & \mu \mathrm{g} / \mathrm{L} & 16^{\mathrm{a}} & \text { NA } \\ & \text { Lead } & \mu \mathrm{g} / \mathrm{L} & 16^{\mathrm{a}} & \text { NA }\end{array}$

a Duplicate samples of lead.

b $\mathrm{NA}=$ not analyzed.

Table 2. Constituents Exceeding the Primary Drinking Water Standards at the Par Pond Sewage Sludge Application Site

$\begin{array}{lllll}\text { Well } & \text { Constituent } & \text { Unit } & 1092 & \underline{2092} \\ N^{a} & \text { None } & N & N & N\end{array}$

a $\mathbf{N}=$ not applicable. 
Table 3. Constituents Exceeding Half the Primary Drinking Water Standards or Other Flag 1 or Flag 2 Criteria at the K-Area Sewage Sludge Application Site

$\begin{array}{lllll}\text { Well } & \text { Constituent } & \text { Unit } & 2092 & \text { Flag } \\ N^{*} & \text { None } & \text { N } & \text { N } & \text { N }\end{array}$

- $\mathbf{N}=$ not applicable.

Table 4. Constituents Exceeding Half the Primary Drinking Mater Standards or Other Flag 1 or Flag 2 Criteria at the Par Pond Sewage Sludge Application Site

\begin{tabular}{lllll} 
Well & Constituent & Unit & $\underline{2092}$ & Flag \\
None & $N$ & $N$ & $N$ \\
\hline$N=$ not applicable. & & &
\end{tabular}


Table 5. Groundwater Monitoring Results for Individual Wells at the K-Area Sewage Sludge Application Site

WELL KSS 1D

\begin{tabular}{|c|c|c|c|c|c|}
\hline SRS Coord. & Lat/Longitude & Screen Zone Elevation & Top of Casing & Casing & Formation \\
\hline $\begin{array}{l}N 47758.9 \\
\text { E40219.1 }\end{array}$ & $\begin{array}{l}33.197023^{\circ} \mathrm{N} \\
81.653674^{\circ} \mathrm{W}\end{array}$ & $177.5-157.4 \mathrm{ft} \mathrm{msl}$ & $229.8 \mathrm{ft} \mathrm{msl}$ & 4" PVC & \\
\hline
\end{tabular}

FIELD MEASUREMENTS

Sample date: $C 5 / 28 / 92$

Depth to wate- $53.96 \mathrm{ft}(16.45 \mathrm{~m})$ below TOC

Water elevatic.l: $175.84 \mathrm{ft}(53.60 \mathrm{~m}) \mathrm{msl}$

Sp. conductance: $50 \mu \mathrm{S} / \mathrm{cm}$

Water evacuated before sampling: $48 \mathrm{gal}$

\section{LABORATORY ANALYSES}

NE Analvte

$\because \mathrm{pH}$

- Specific conductance

Specific conductance

Acenaphthene

Acenaphthylene

Anthracene

Benzidine

Benzoljlanthracsne

Benxclolfluorantl:tne

Benzoik fllcoranthene

Eenzolg,h,i]perylene

Benzolaloyrene

Bis/2-chtsroethoxy) methane

Bis(2-chloroethyl) ether

Bis(2-chloroisopropyl) ether

Bis (2,ethylhexyl) phthalate

4 Bromophenyl phenyl ether

Butylbenzyl phthalate

Chloride

para-Chloro-meta-cresol

2-Chloronaphthalene

2-Chlorophenol

4-Chlorophenyl phenyl ether

Chrysene

Dibenzla,h]anthracene

Di-n-butyl phthalate

1,2-Dichlorobenzene

1,3-Dichlorobenzene

1,4-Dichlorobenzene

3,3'-Dichlorobenzidine

2,4-Dichlorophenol

Diethyl phthalate

2,4-Dimethyl phenol

Dimethyl phthalate

4,6-Dinitro-ortho-cresol
Time: 13:00

pH: 5.7

Alkalinity: $8 \mathrm{mg} / \mathrm{L}$

Water temperature: $20.3^{\circ} \mathrm{C}$

\begin{tabular}{|c|c|c|}
\hline Result & Unit & Flag \\
\hline $\begin{aligned} & 6.5 \\
& 6.5 \\
& 47 \\
& 47 \\
&< 10 \\
&< 10 \\
&<10 \\
&<50 \\
&<10 \\
&<10 \\
&<10 \\
&<10 \\
&<10 \\
&<10 \\
&<10 \\
&<10 \\
&<18 \\
&<10 \\
&<10 \\
& 3300 \\
&<10 \\
&<10 \\
&<10 \\
&<10 \\
&<10 \\
&<10 \\
&<10 \\
& 210 \\
&<10 \\
&<10 \\
&<10 \\
&<10 \\
&<10 \\
&<10 \\
&<10 \\
&<10 \\
&<10 \\
&<50\end{aligned}$ & $\begin{array}{l}p H \\
p H \\
\mu S / l \\
\mu S / l \\
\mu g / L \\
\mu g / L \\
\mu g / L \\
\mu g / L \\
\mu g / L \\
\mu g / L \\
\mu g / L \\
\mu g / L \\
\mu g / L \\
\mu g / L \\
\mu g / L \\
\mu g / L \\
\mu g / L \\
\mu g / L \\
\mu g / L \\
\mu g / L \\
\mu g / L \\
\mu g / L \\
\mu g / L \\
\mu g / L \\
\mu g / L \\
\mu g / L \\
\mu g / L \\
\mu g / L \\
\mu g / L \\
\mu g / L \\
\mu g / L \\
\mu g / L \\
\mu g / L \\
\mu g / L \\
\mu g / L \\
\mu g / L\end{array}$ & $\begin{array}{l}0 \\
0 \\
0 \\
0 \\
0 \\
0 \\
0 \\
0 \\
0 \\
0 \\
0 \\
0 \\
0 \\
0 \\
0 \\
0 \\
0 \\
0 \\
0 \\
0 \\
0 \\
0 \\
0 \\
0 \\
0 \\
0 \\
0 \\
0 \\
0 \\
0 \\
0 \\
0 \\
0 \\
0 \\
0 \\
0\end{array}$ \\
\hline
\end{tabular}

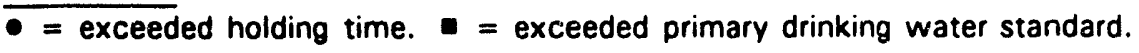


WELL KSS 1D collected on 05/28/92, laboratory analyses (cont.)

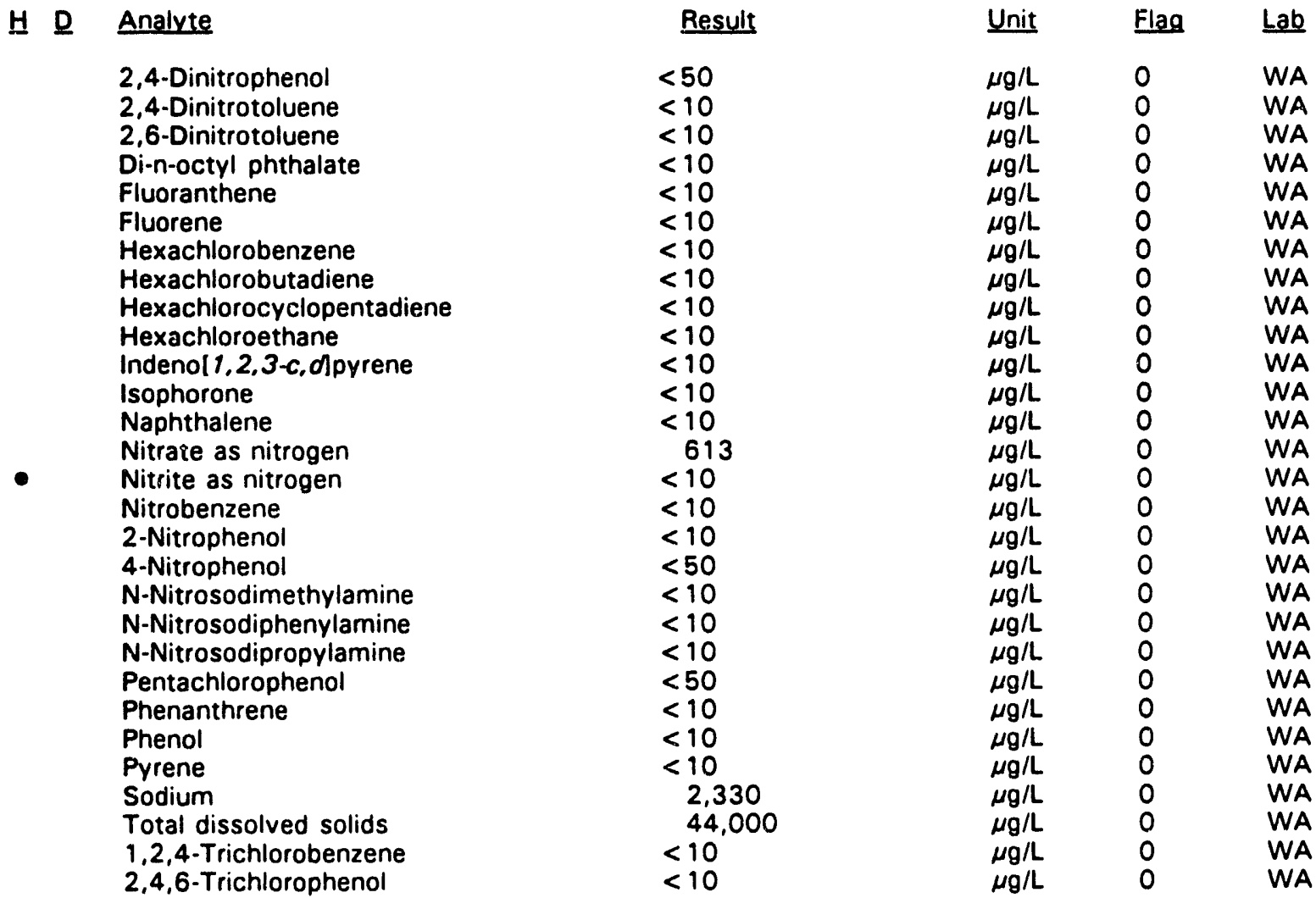

WELL KSS 2D

$\begin{array}{llllll}\text { SRS Coord. } & \text { Lat/Longitude } & \text { Screen Zone Elevation } & \text { Top of Casing } & \text { Casing } & \text { Formation } \\ \text { N46803.8 } & 33.195266^{\circ} \mathrm{N} & 164.7-144.6 \mathrm{ft} \mathrm{msl} & 192.3 \mathrm{ft} \mathrm{msl} & 4^{\mathrm{n}} \mathrm{PVC} & \text { Water table } \\ \text { E40437.0 } & 81.651247^{\circ} \mathrm{W} & & & & \end{array}$

\section{FIELD MEASUREMENTS}

Sample date: $05 / 28 / 92$

Depth to water: $27.06 \mathrm{ft}(8.25 \mathrm{~m})$ below TOC

Water elevation: $165.24 \mathrm{ft}(50.37 \mathrm{~m}) \mathrm{msl}$

Sp. conductance: $23 \mu \mathrm{S} / \mathrm{cm}$

Water evacuated before sampling: $54 \mathrm{gal}$

Time: $12: 00$

pH: 5.2

Alkalinity: $5 \mathrm{mg} / \mathrm{L}$

Water temperature: $19.8^{\circ} \mathrm{C}$

\section{LABORATORY ANALYSES}

H D Analyte

pH

Specific conductance

Acenaphthene

Acenaphthylene

Anthracene

Benzidine
Result

$$
6.3
$$

18

$<10$

$<10$

$<10$

$<50$

Unit
$\mathrm{pH}$
$\mu S / \mathrm{cm}$
$\mu g / L$
$\mu g / L$
$\mu g / L$
$\mu g / L$

Flag $\quad$ Lab

0

WA

0 WA

0 WA

WA

WA

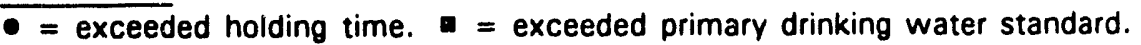


WELL KSS 2D collected on 05/28/92, laboratory analyses (cont.)

H D Analyte

Benzolalanthracene

Benzolblfluoranthene

Benzolk]fluoranthene

Benzolg, $h, i$ perylene

Benzola]pyrene

Bis(2-chloroethoxy) methane

Bis(2-chloroethyl) ether

Bis(2-chloroisopropyl) ether

Bis (2-ethylhexyl) phthalate

4-Bromophenyl phenyl ether

Butylbenzyl phthalate

Chloride

para-Chloro-meta-cresol

2-Chloronaphthalene

2-Chlorophenol

4-Chlorophenyl phenyl ether

Chrysene

Dibenzla, hlanthracene

Di-n-butyl phthalate

1,2-Dichlorobenzene

1,3-Dichlorobenzene

1,4-Dichlorobenzene

3,3'-Dichlorobenzidine

2,4-Dichlorophenol

Diethyl phthalate

2,4-Dimethyl phenol

Dimethyl phthalate

4.6-Dinitro-ortho-cresol

2,4-Dinitrophenol

2,4-Dinitrotoluene

2,6-Dinitrotoluene

Di-n-octyl phthalate

Fluoranthene

Fluorene

Hexachlorobenzene

Hexachlorobutadiene

Hexachlorocyclopentadiene

Hexachloroethane

Indenol 1,2,3-c, olpyrene

Isophorone

Naphthalene

Nitrate as nitrogen

Nitrite as nitrogen

Nitrobenzene

2-Nitrophenol

4-Nitrophenol

N-Nitrosodimethylamine

N-Nitrosodiphenylamine

N-Nitrosodipropylamine

Pentachlorophenol

Phenanthrene

Phenol

Pyrene

Sodium

Total dissolved solids

1,2,4-Trichlorobenzene

2,4,6-Trichlorophenol
Result

$<10$

$<10$

$<10$

$<10$

$<10$

$<10$

$<10$

$<10$

14

$<10$

$<10$

2,930

$<10$

$<10$

$<10$

$<10$

$<10$

$<10$

1.7

$<10$

$<10$

$<10$

$<20$

$<10$

$<10$

$<10$

$<10$

$<50$

$<50$

$<10$

$<10$

$<10$

$<10$

$<10$

$<10$

$<10$

$<10$

$<10$

$<10$

$<10$

$<10$

555

$<10$

$<10$

$<10$

$<50$

$<10$

$<10$

$<10$

$<50$

$<10$

$<10$

$<10$

1,780

19,000

$<10$

$<10$

\begin{tabular}{|c|c|c|}
\hline Unit & Flag & $\underline{\text { Lab }}$ \\
\hline$\mu g / L$ & 0 & WA \\
\hline$\mu g / L$ & 0 & WA \\
\hline$\mu \mathrm{g} / \mathrm{L}$ & 0 & WA \\
\hline$\mu \mathrm{g} / \mathrm{L}$ & 0 & WA \\
\hline$\mu \mathrm{g} / \mathrm{L}$ & 0 & WA \\
\hline$\mu \mathrm{g} / \mathrm{L}$ & 0 & WA \\
\hline$\mu \mathrm{g} / \mathrm{L}$ & 0 & WA \\
\hline$\mu g / L$ & 0 & WA \\
\hline$\mu g / L$ & 0 & WA \\
\hline$\mu g / L$ & 0 & WA \\
\hline$\mu g / L$ & 0 & WA \\
\hline$\mu g / L$ & 0 & WA \\
\hline$\mu g / L$ & 0 & WA \\
\hline$\mu g / L$ & 0 & WA \\
\hline$\mu \mathrm{g} / \mathrm{L}$ & 0 & WA \\
\hline$\mu g / L$ & 0 & WA \\
\hline$\mu g / L$ & 0 & WA \\
\hline$\mu g / L$ & 0 & WA \\
\hline$\mu g / L$ & 0 & WA \\
\hline$\mu g / L$ & 0 & WA \\
\hline$\mu g / L$ & 0 & WA \\
\hline$\mu g / L$ & 0 & WA \\
\hline$\mu \mathrm{g} / \mathrm{L}$ & 0 & WA \\
\hline$\mu \mathrm{g} / \mathrm{L}$ & 0 & WA \\
\hline$\mu \mathrm{g} / \mathrm{L}$ & 0 & WA \\
\hline$\mu \mathrm{g} / \mathrm{L}$ & 0 & WA \\
\hline$\mu \mathrm{g} / \mathrm{L}$ & 0 & WA \\
\hline$\mu g / L$ & 0 & WA \\
\hline$\mu \mathrm{g} / \mathrm{L}$ & 0 & WA \\
\hline$\mu g / L$ & 0 & WA \\
\hline$\mu g / L$ & 0 & WA \\
\hline$\mu g / L$ & 0 & WA \\
\hline$\mu \mathrm{g} / \mathrm{L}$ & 0 & WA \\
\hline$\mu g / L$ & 0 & WA \\
\hline$\mu \mathrm{g} / \mathrm{L}$ & 0 & WA \\
\hline$\mu g / L$ & 0 & WA \\
\hline$\mu g / L$ & 0 & WA \\
\hline$\mu g / L$ & 0 & WA \\
\hline$\mu g / L$ & 0 & WA \\
\hline$\mu g / L$ & 0 & WA \\
\hline$\mu g / L$ & 0 & WA \\
\hline$\mu g / L$ & 0 & WA \\
\hline$\mu g / L$ & 0 & WA \\
\hline$\mu g / L$ & 0 & WA \\
\hline$\mu g / L$ & 0 & WA \\
\hline$\mu g / L$ & 0 & WA \\
\hline$\mu g / L$ & 0 & WA \\
\hline$\mu g / L$ & 0 & WA \\
\hline$\mu g / L$ & 0 & WA \\
\hline$\mu g / L$ & 0 & WA \\
\hline$\mu g / L$ & 0 & WA \\
\hline$\mu g / L$ & 0 & WA \\
\hline$\mu \mathrm{g} / \mathrm{L}$ & 0 & WA \\
\hline$\mu g / L$ & 0 & WA \\
\hline$\mu g / L$ & 0 & WA \\
\hline$\mu g / L$ & 0 & WA \\
\hline$\mu g / L$ & 0 & WA \\
\hline
\end{tabular}

$\overline{-=\text { exceeded holding time. }}$ = exceeded primary drinking water standard.

K.Area and Par Pord Sewage Sludge D.7 
WELL KSS 3D

$\begin{array}{lllllll}\text { SRS Coord. Lat/Longitude } & \text { Screen Zone Elevation } & \text { Top of Casing } & \text { Casing } & \text { Formation } \\ \text { N46644.3 } & 33.195420^{\circ} \mathrm{N} & 159.3-139.3 \mathrm{ft} \mathrm{msl} & 185.2 \mathrm{ft} \mathrm{msl} & \text { 4" PVC } & \text { Water table } \\ \text { E40748.0 } & 81.650120^{\circ} \mathrm{W} & & & \end{array}$

\section{FIELD MEASUREMENTS}

Sample date: 05/28/92

Depth to water: $20.67 \mathrm{ft}(6.30 \mathrm{~m})$ below TOC

Water elevation: $164.53 \mathrm{ft}(50.15 \mathrm{~m}) \mathrm{msl}$

Sp. conductance: $32 \mu \mathrm{S} / \mathrm{cm}$

Water evacuated before sampling: $66 \mathrm{gal}$

Time: $11: 15$

pH: 5.6

Alkalinity: $9 \mathrm{mg} / \mathrm{L}$

Water temperature: $18.6^{\circ} \mathrm{C}$

LABORATORY ANALYSES

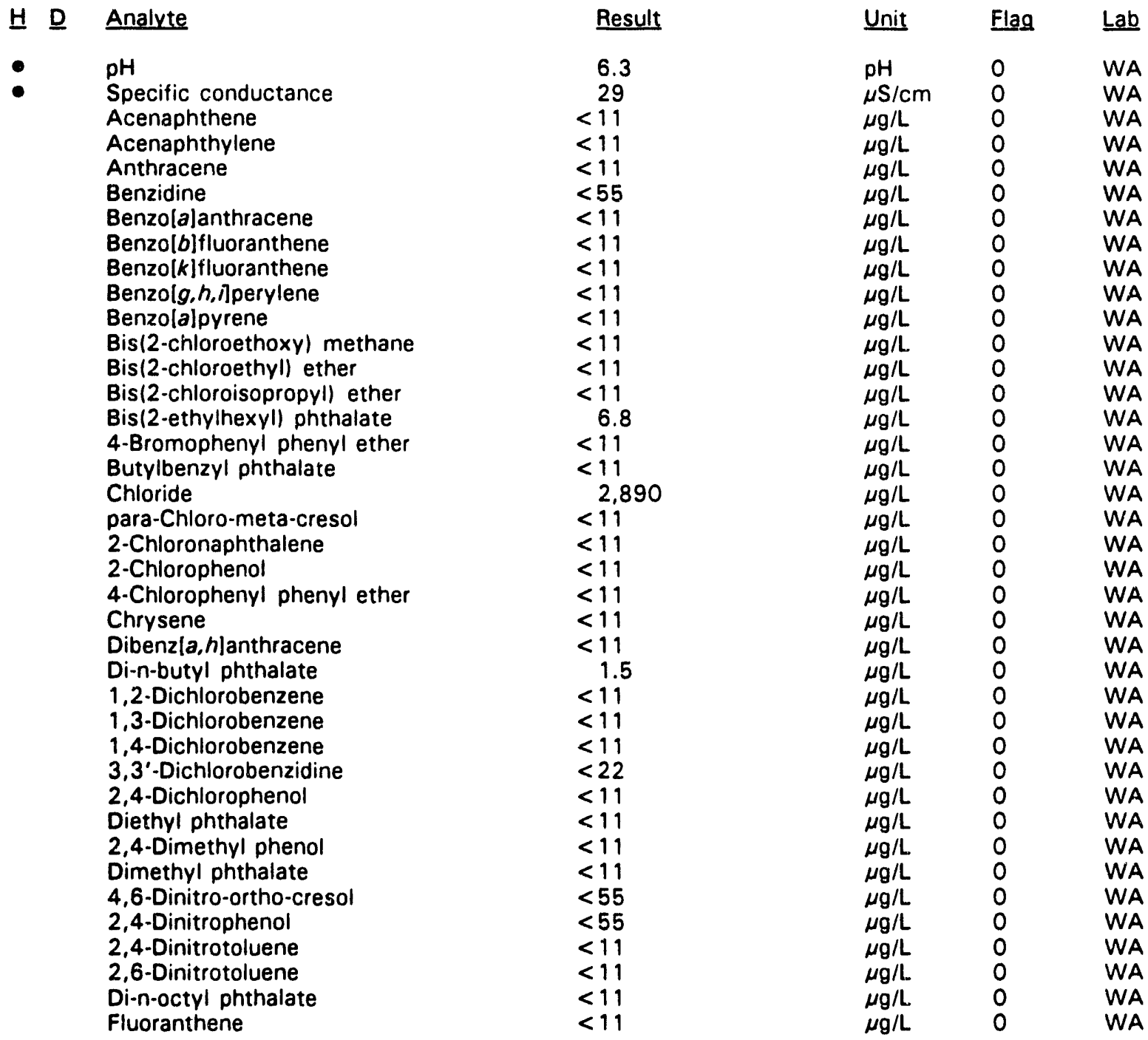

\footnotetext{
$\overline{- \text { exceeded holding time. }}$ = exceeded primary drinking water standard.
} 
WELL KSS 3D collected on $05 / 28 / 92$, laboratory analyses (cont.)

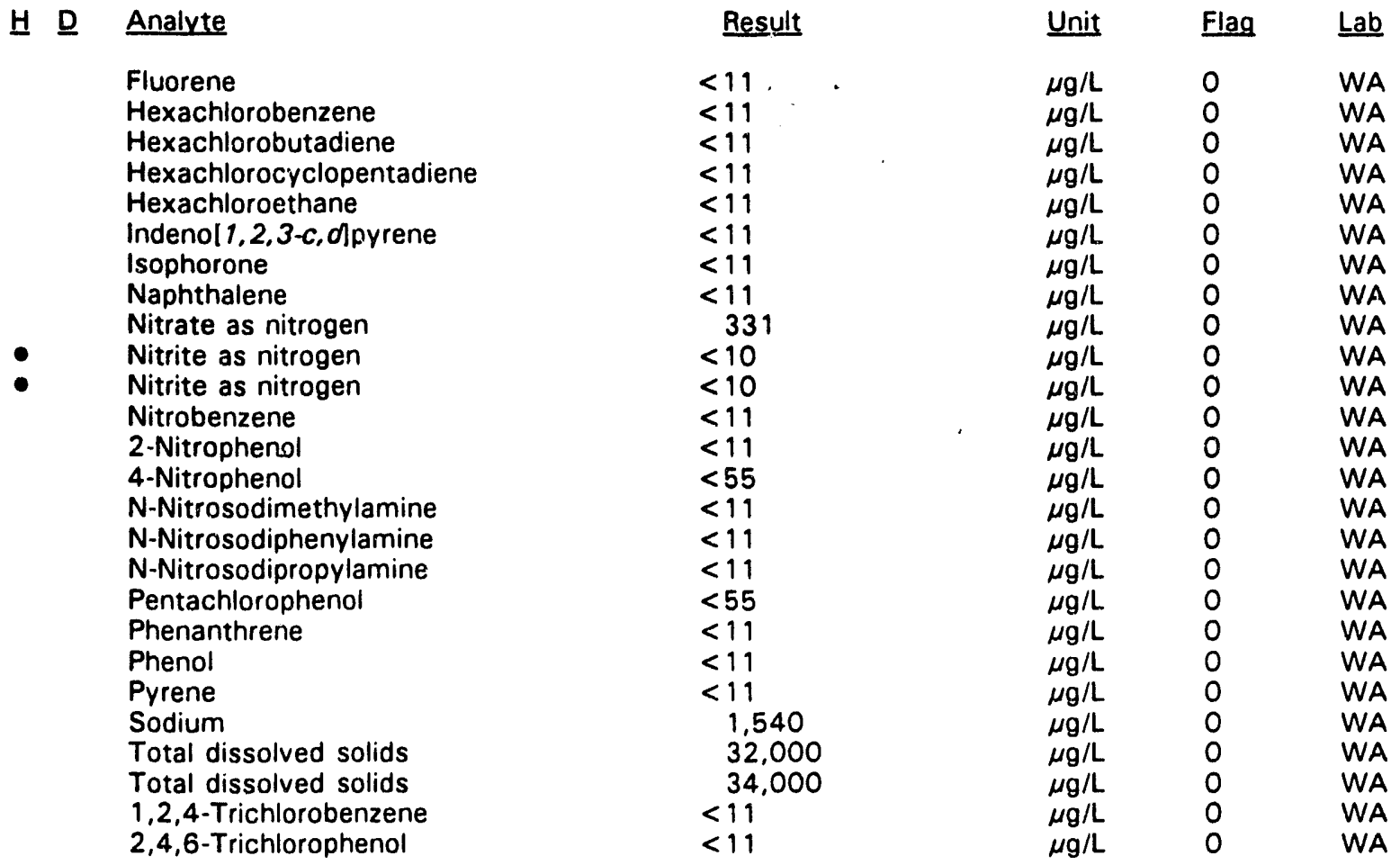

$\overline{- \text { exceeded holding time. }} \mathbf{\square}=$ exceeded primary drinking water standard. 


\section{Table 6. Groundwater Monitoring Resuits for Individual Wells at the Par Pond Sewage Sludge Application Site}

\section{WELL PSS 1D}

\begin{tabular}{lllllll} 
SRS Coord. & Lat/Longitude & \multicolumn{1}{c}{ Screen Zone Elevation } & Top of Casing & Casing & Formation \\
N37298.4 & $33.231837^{\circ} \mathrm{N}$ & $202.1-182.1 \mathrm{ft} \mathrm{msl}$ & $219.6 \mathrm{ft} \mathrm{msl}$ & 4" PVC & Water table \\
E75773.3 & $81.539790^{\circ} \mathrm{W}$ & & &
\end{tabular}

\section{FIELD MEASUREMENTS}

Sample date: 06/17/92

Depth to water: $24.45 \mathrm{ft}(7.45 \mathrm{~m})$ below TOC

Water elevation: $195.15 \mathrm{ft}(59.48 \mathrm{~m}) \mathrm{ms}$ l

Sp. conductance: $13 \mu \mathrm{S} / \mathrm{cm}$

Water evacuated before sampling: $34 \mathrm{gal}$

\section{LABORATORY ANALYSES}

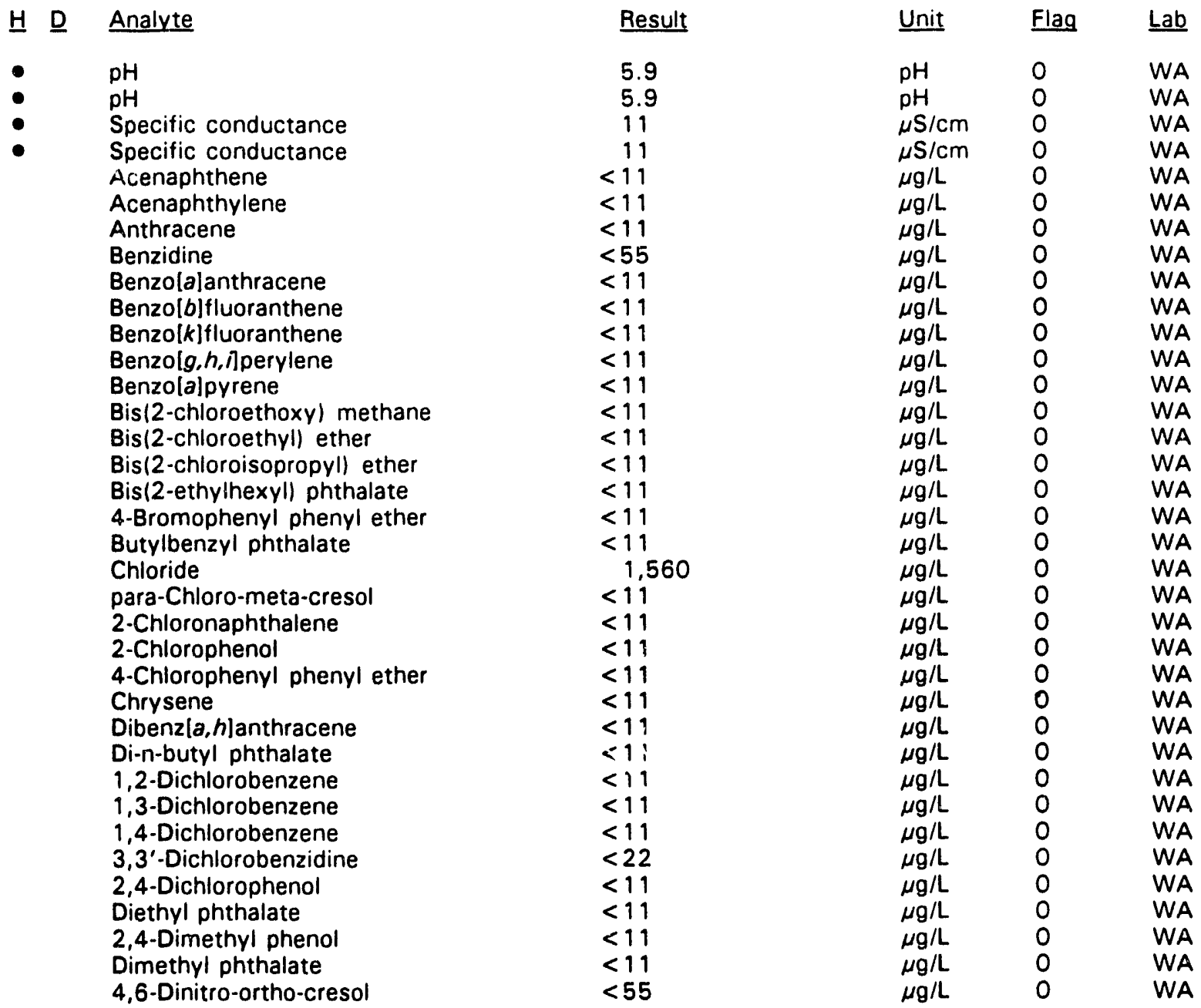

\footnotetext{
$\overline{-=}$ exceeded holding time. $=$ exceeded primary drinking water standard.
}

K-Area and Par Pond Sewage Sludge

D.10

Time: 10:00

pH: 4.7

Alkalinity: $1 \mathrm{mg} / \mathrm{L}$

Water temperature: $19.9^{\circ} \mathrm{C}$ 
WELL PSS 1D collected on 06/17/92, laboratory analyses (cont.)

H $\underline{\text { Analyte }}$

2,4-Dinitrophenol
2,4-Dinitrotoluene
2,6-Dinitrotoluene
Di-n-octyl phthalate
Fluoranthene
Fluorene
Hexachlorobenzene
Hexachlorobutadiene
Hexachlorocyclopentadiene
Hexachloroethane
Indenol1,2,3-c, Olpyrene
Isophorone
Naphthalene
Nitrate as nitrogen
Nitrite as nitrogen
Nitrobenzene
2-Nitrophenol
4-Nitrophenol
N-Nitrosodimethylamine
N-Nitrosodiphenylamine
N-Nitrosodipropylamine
Pentachlorophenol
Phenanthrene
Phenol
Pyrene
Sodium
Total dissolved solids
1,2,4-Trichlorobenzene
2,4,6-Trichlorophenol

\section{Result}

$<55$

$<11$

$<11$

$<11$

$<11$

$<11$

$<19$

$<11$

$<11$

$<11$

$<11$

$<11$

$<11$

505

$<10$

$<11$

$<11$

$<55$

$<11$

$<11$

$<11$

$<55$

$<11$

$<11$

$<11$

673

25,000

$<11$

$<11$

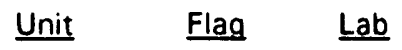

$\mu g / L \quad 0 \quad$ WA

$\mu g / L \quad 0 \quad$ WA

$\mu g / L \quad 0 \quad$ WA

$\mu g / L \quad 0 \quad W A$

$\mu g / L \quad 0 \quad$ WA

$\mu g / L \quad 0 \quad$ WA

$\mu \mathrm{g} / \mathrm{L} \quad \mathrm{O}$ WA

$\mu g / L \quad 0 \quad$ WA

$\mu g / L \quad 0 \quad W A$

$\mu g / L \quad 0 \quad$ WA

$\mu g / L \quad 0 \quad W A$

$\mu g / L \quad 0 \quad$ WA

$\mu g / L \quad 0 \quad W A$

$\mu g / L \quad 0 \quad W A$

WA

$\mu g / L \quad 0 \quad$ WA

$\mu g / L$

$\mu g / L \quad 0 \quad W A$

$\begin{array}{lll}\mu g / L & 0 & W A\end{array}$

$\mu g / L \quad 0 \quad$ WA

$\mu g / L \quad 0 \quad W A$

$\mu g / L \quad 0 \quad$ WA

$\mu g / L \quad 0 \quad W A$

$\mu g / L \quad 0 \quad W A$

$\mu g / L \quad 0 \quad$ WA

$\mu g / L \quad 0 \quad$ WA

$\mu g / L \quad 0 \quad W A$

$\mu g / L \quad 0 \quad W A$

$\begin{array}{lll}\mu \mathrm{g} / \mathrm{L} & 0 & \text { WA }\end{array}$

\section{WELL PSS 2D}

\begin{tabular}{|c|c|c|c|c|c|}
\hline SRS Coord. & Lat/Longitude & Screen Zone Elevation & Top of Casing & Casing & Formation \\
\hline $\begin{array}{l}N 36037.9 \\
E 75910.1\end{array}$ & $\begin{array}{l}33.229270^{\circ} \mathrm{N} \\
81.536993^{\circ} \mathrm{W}\end{array}$ & $197.1-177.1 \mathrm{ft} \mathrm{msl}$ & $228.7 \mathrm{ft} \mathrm{msl}$ & 4" PVC & table \\
\hline
\end{tabular}

\section{FIELD MEASUREMENTS}

Sample date: 06/17/92

Depth to water: $35.38 \mathrm{ft}(10.78 \mathrm{~m})$ below TOC Water elevation: $193.32 \mathrm{ft}(58.92 \mathrm{~m}) \mathrm{ms}$

Sp. conductance: $15 \mu \mathrm{S} / \mathrm{cm}$

Water evacuated before sampling: $43 \mathrm{gal}$

\section{LABORATORY ANALYSES}

Analyte

$\mathrm{pH}$

Specific conductance

Acenaphthene

Acenaphthylene
Time: $9: 25$

$\mathrm{pH}: 4.5$

Alkalinity: $0 \mathrm{mg} / \mathrm{L}$

Water temperature: $19.3^{\circ} \mathrm{C}$ 
WELL. PSS 2D collected on 06/17/92, laboratory analyses (cont.)

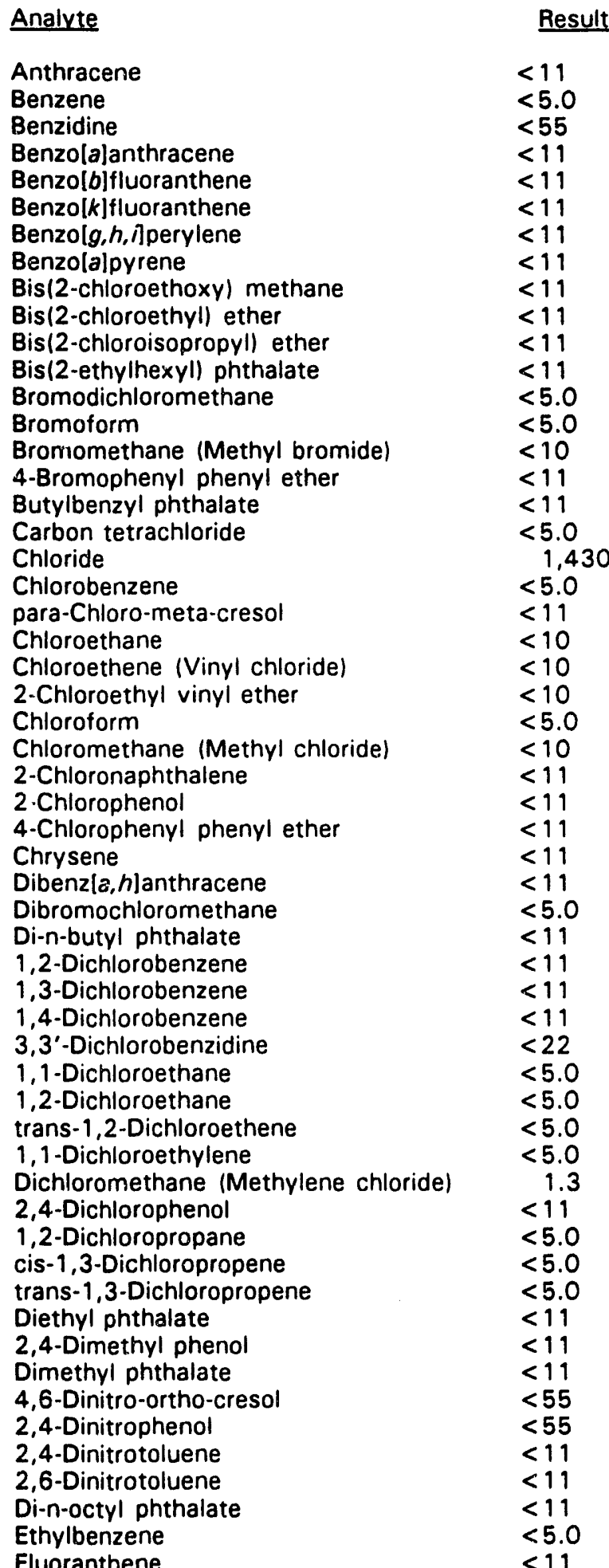

\begin{tabular}{|c|c|}
\hline Unit & Flag \\
\hline$\mu g / L$ & 0 \\
\hline$\mu \mathrm{g} / \mathrm{L}$ & 0 \\
\hline$\mu \mathrm{g} / \mathrm{L}$ & 0 \\
\hline$\mu g / L$ & 0 \\
\hline$\mu g / L$ & 0 \\
\hline$\mu g / L$ & 0 \\
\hline$\mu g / L$ & 0 \\
\hline$\mu \mathrm{g} / \mathrm{L}$ & 0 \\
\hline$\mu g / L$ & 0 \\
\hline$\mu g / L$ & 0 \\
\hline$\mu g / L$ & 0 \\
\hline$\mu g / L$ & 0 \\
\hline$\mu g / L$ & 0 \\
\hline$\mu g / L$ & 0 \\
\hline$\mu g / L$ & 0 \\
\hline$\mu g / L$ & 0 \\
\hline$\mu g / L$ & 0 \\
\hline$\mu \mathrm{g} / \mathrm{L}$ & 0 \\
\hline$\mu \mathrm{g} / \mathrm{L}$ & 0 \\
\hline$\mu \mathrm{g} / \mathrm{L}$ & 0 \\
\hline$\mu \mathrm{g} / \mathrm{L}$ & 0 \\
\hline$\mu \mathrm{g} / \mathrm{L}$ & 0 \\
\hline$\mu \mathrm{g} / \mathrm{L}$ & 0 \\
\hline$\mu \mathrm{g} / \mathrm{L}$ & 0 \\
\hline$\mu g / L$ & 0 \\
\hline$\mu \mathrm{g} / \mathrm{L}$ & 0 \\
\hline$\mu \mathrm{g} / \mathrm{L}$ & 0 \\
\hline$\mu g / L$ & 0 \\
\hline$\mu g / L$ & 0 \\
\hline$\mu \mathrm{g} / \mathrm{L}$ & 0 \\
\hline$\mu g / L$ & 0 \\
\hline$\mu \mathrm{g} / \mathrm{L}$ & 0 \\
\hline$\mu g / L$ & 0 \\
\hline$\mu \mathrm{g} / \mathrm{L}$ & 0 \\
\hline$\mu \mathrm{g} / \mathrm{L}$ & 0 \\
\hline$\mu \mathrm{g} / \mathrm{L}$ & 0 \\
\hline$\mu \mathrm{g} / \mathrm{L}$ & 0 \\
\hline$\mu g / L$ & 0 \\
\hline$\mu \mathrm{g} / \mathrm{L}$ & 0 \\
\hline$\mu g / L$ & 0 \\
\hline$\mu g / L$ & 0 \\
\hline$\mu g / L$ & 0 \\
\hline$\mu g / L$ & 0 \\
\hline$\mu g / L$ & 0 \\
\hline$\mu \mathrm{g} / \mathrm{L}$ & 0 \\
\hline$\mu \mathrm{g} / \mathrm{L}$ & 0 \\
\hline$\mu \mathrm{g} / \mathrm{L}$ & 0 \\
\hline$\mu \mathrm{g} / \mathrm{L}$ & 0 \\
\hline$\mu g / L$ & 0 \\
\hline$\mu \mathrm{g} / \mathrm{L}$ & 0 \\
\hline$\mu \mathrm{g} / \mathrm{L}$ & 0 \\
\hline$\mu g / L$ & 0 \\
\hline$\mu \mathrm{g} / \mathrm{L}$ & 0 \\
\hline$\mu g / L$ & 0 \\
\hline$\mu g / L$ & 0 \\
\hline & \\
\hline
\end{tabular}

$\overline{0}$ = exceeded holding time. $=$ exceeded primary drinking water standard. 
WELL PSS 2D collected on 06/17/92, laboratory analyses (cont.)

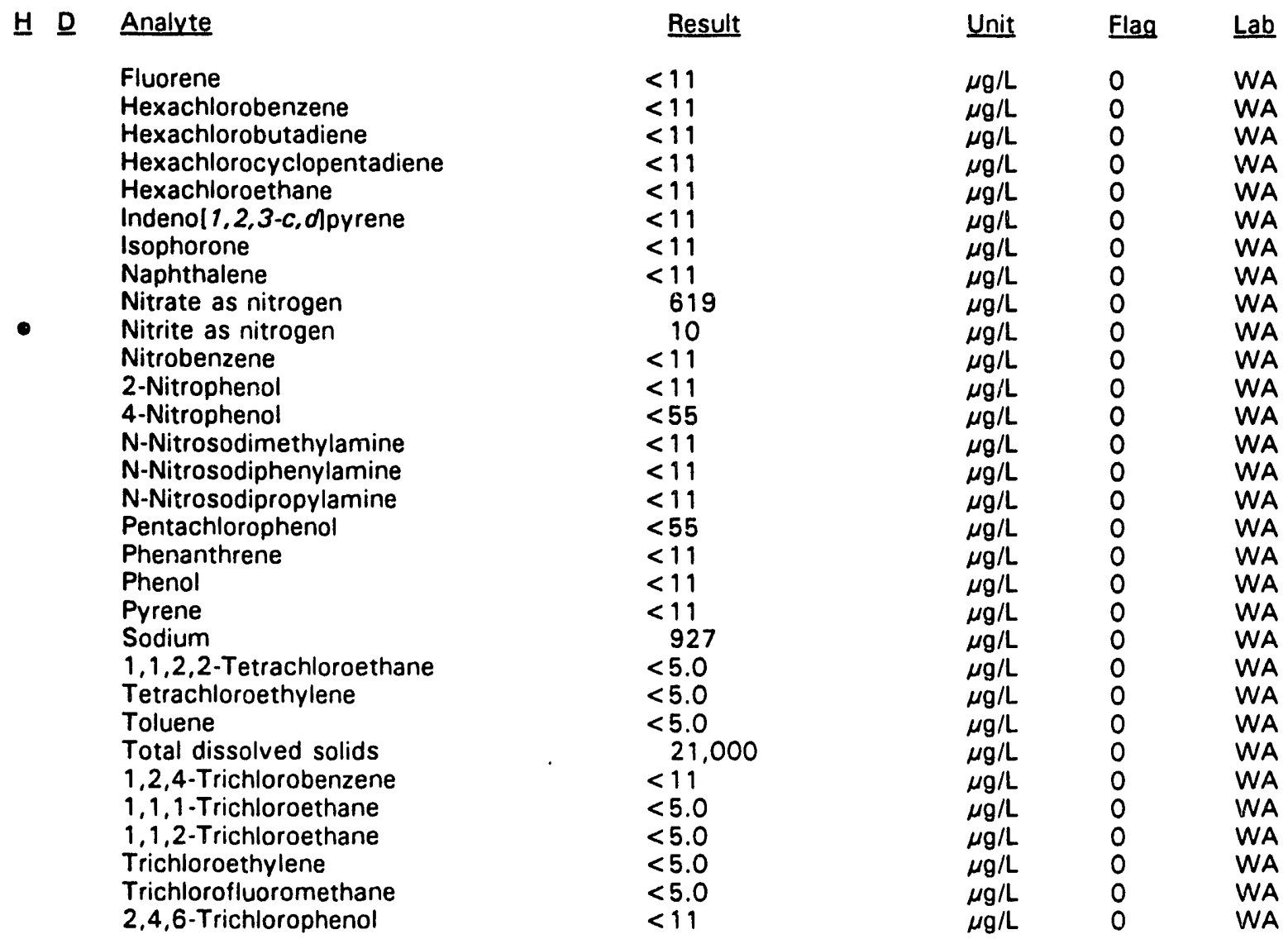

WELL PSS 3D

\begin{tabular}{|c|c|c|c|c|c|}
\hline SRS Coord. & Lat/Longitude & $\underline{\text { Screen Zone Elevation }}$ & Top of Casing & Casing & Formation \\
\hline $\begin{array}{l}\text { N35974.1 } \\
\text { E76138.7 }\end{array}$ & $\begin{array}{l}33.229501^{\circ} \mathrm{N} \\
81.536268^{\circ} \mathrm{W}\end{array}$ & $198.5-178.5 \mathrm{ft} \mathrm{msl}$ & $234.0 \mathrm{ft} \mathrm{msl}$ & 4" PVC & \\
\hline
\end{tabular}

\section{FIELD MEASUREMENTS}

Sample date: 06/17/92

Depth to water: Not available

Time: $14: 15$

Water elevation: Not available

$\mathrm{pH}: 4.8$

Sp. conductance: $9 \mu \mathrm{S} / \mathrm{cm}$

Alkalinity: $1 \mathrm{mg} / \mathrm{L}$

Water evacuated before sampling: $6 \mathrm{gal}$

Water temperature: $19.9^{\circ} \mathrm{C}$

The well went dry during purging.

\section{LABORATORY ANALYSES}

$\begin{array}{llllll}\text { H } & \text { Analyte } & \text { Result } & \text { Unit } & \text { Flag } & \text { Lab } \\ \text { - } & \text { pH } & 5.4 & \text { pH } & 0 & \text { WA } \\ \text { Specific conductance } & 7.9 & \mu \mathrm{S} / \mathrm{cm} & 0 & \text { WA }\end{array}$

$\overline{-}=$ exceeded holding time. $=$ exceeded primary drinking water standard. 
WELL PSS 3D collected on 06/17/92, laboratory analyses (cont.)

\section{H D Analyte}

Acenaphthene

Acenaphthylene

Anthracens

Benzidine

Benzolalanthracene

Benzolb]fluoranthene

Benzolk]fluoranthene

Benzolg, $h, i]$ perylene

Benzolalpyrene

Bis(2-chloroethoxy) methane

Bis(2-chloroethyl) ether

Bis(2-chloroisopropyl) ether

Bis(2-ethylhexyl) phthalate

4-Bromophenyl phenyl ether

Butylbenzyl phthalate

Chloride

para-Chloro-meta-cresol

2-Chloronaphthalene

2-Chlorophenol

4-Chlorophenyl phenyl ether

Chrysene

Dibenz $[a, h]$ anthracene

Di-n-butyl phthalate

1,2-Dichlorobenzene

1,3-Dichlorobenzene

1,4-Dichlorobenzene

3.3'-Dichlorobenzidine

2,4-Dichlorophenol

Diethyl phthalate

2,4-Dimethyl phenol

Dimethyl phthalate

4,6-Dinitro-ortho-cresol

2,4-Dinitrophenol

2,4-Dinitrotoluene

2,6-Dinitrotoluene

Di-n-octyl phthalate

Fluoranthene

Fluorene

Hexachlorobenzene

Hexachlorobutadiene

Hexachlorocyclopentadiene

Hexachloroethane

Indenol 1,2,3-c, d]pyrene

Isophorone

Naphthalene

Nitrate as nitrogen

- Nitrite as nitrogen

Nitrobenzene

2-Nitrophenol

4-Nitrophenol

N-Nitrosodimethylamine

N-Nitrosodiphenylamine

N-Nitrosodipropylamine

Pentachlorophenol

Phenanthrene

Phenol

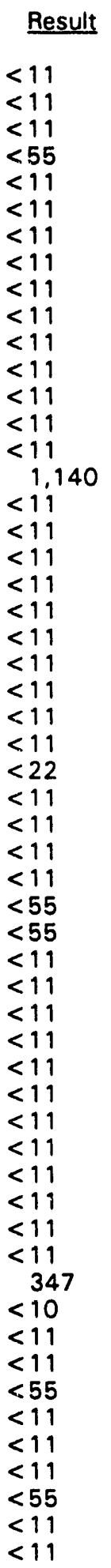

$\overline{- \text { = exceeded holding time. }}$ = exceeded primary drinking water standard.

\begin{tabular}{lll} 
Unit & Flag & Lab \\
\hline$\mu g / L$ & 0 & WA \\
$\mu g / L$ & 0 & WA \\
$\mu g / L$ & 0 & WA \\
$\mu g / L$ & 0 & WA \\
$\mu g / L$ & 0 & WA \\
$\mu g / L$ & 0 & WA \\
$\mu g / L$ & 0 & WA \\
$\mu g / L$ & 0 & WA \\
$\mu g / L$ & 0 & WA \\
$\mu g / L$ & 0 & WA \\
$\mu g / L$ & 0 & WA \\
$\mu g / L$ & 0 & WA \\
$\mu g / L$ & 0 & WA \\
$\mu g / L$ & 0 & WA \\
$\mu g / L$ & 0 & WA \\
$\mu g / L$ & 0 & WA \\
$\mu g / L$ & 0 & WA \\
$\mu g / L$ & 0 & WA \\
$\mu g / L$ & 0 & WA \\
$\mu g / L$ & 0 & WA \\
$\mu g / L$ & 0 & WA \\
$\mu g / L$ & 0 & WA \\
$\mu g / L$ & 0 & WA \\
$\mu g / L$ & 0 & WA \\
$\mu g / L$ & 0 & WA \\
$\mu g / L$ & 0 & WA \\
$\mu g / L$ & 0 & WA \\
$\mu g / L$ & 0 & WA \\
$\mu g / L$ & 0 & WA \\
$\mu g / L$ & 0 & WA \\
$\mu g / L$ & 0 & WA \\
$\mu g / L$ & 0 & WA \\
$\mu g / L$ & 0 & WA \\
$\mu g / L$ & 0 & WA \\
$\mu g / L$ & 0 & WA \\
$\mu g / L$ & 0 & WA \\
$\mu g / L$ & 0 & WA \\
$\mu g / L$ & 0 & WA \\
$\mu g / L$ & 0 & WA \\
$\mu g / L$ & 0 & WA \\
$\mu g / L$ & 0 & WA \\
$\mu g / L$ & 0 & WA \\
$\mu g / L$ & 0 & WA \\
$\mu g / L$ & 0 & WA \\
$\mu g / L$ & 0 & WA \\
$\mu g / L$ & 0 & WA \\
$\mu g / L$ & 0 & WA \\
$\mu g / L$ & 0 & WA \\
$\mu g / L$ & 0 & WA \\
$\mu g / L$ & 0 & WA \\
$\mu g / L$ & 0 & WA \\
$\mu g / L$ & 0 & WA \\
$\mu g / L$ & 0 & WA \\
$\mu g / L$ & 0 & WA \\
$\mu g / L$ & 0 & WA \\
$\mu g / L$ & 0 & WA \\
& &
\end{tabular}

K-Area and Par Pond Sewage Sludge 
WELL PSS 3D collected on 06/17/92, laboratory analyses (cont.)

H D $\quad$ Analyte

Pyrene
Sodium
Total dissolved solids
1,2,4-Trichlorobenzene
2,4,6-Trichlorophenol

$\begin{aligned} & \text { Result } \\ < & 11 \\ & 651 \\ & 25,000 \\ < & 11 \\ < & 11\end{aligned}$

$\begin{array}{lll}\text { Unit } & \text { Flag } & \text { Lab } \\ \mu \mathrm{g} / \mathrm{L} & 0 & \text { WA } \\ \mu \mathrm{g} / \mathrm{L} & 0 & \text { WA } \\ \mu \mathrm{g} / \mathrm{L} & 0 & \text { WA } \\ \mu \mathrm{g} / \mathrm{L} & 0 & \text { WA } \\ \mu \mathrm{g} / \mathrm{L} & 0 & \text { WA }\end{array}$

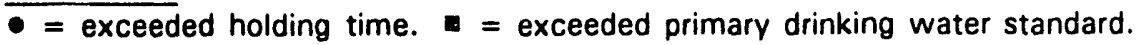



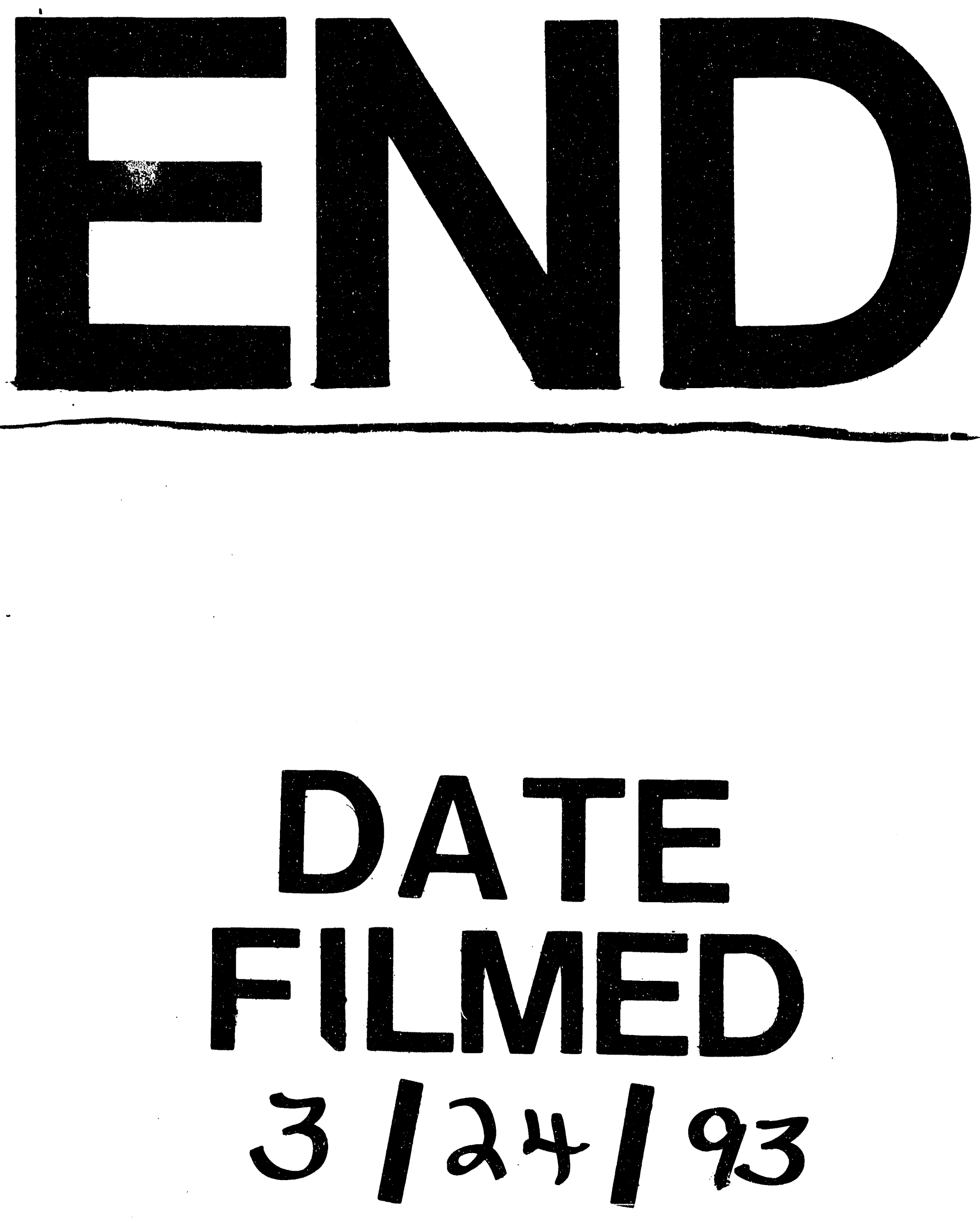
